\title{
Bilateral bargaining with one-sided uncertain reserve prices
}

\author{
Bo An • Nicola Gatti • Victor Lesser
}

Published online: 24 May 2012

(C) The Author(s) 2012

\begin{abstract}
The problem of finding agents' rational strategies in bargaining with incomplete information is well known to be challenging. The literature provides a collection of results for very narrow uncertainty settings, but no generally applicable algorithm. This lack has led researchers to develop heuristic approaches in an attempt to find outcomes that, even if not being of equilibrium, are mutually satisfactory. In the present paper, we focus on the principal bargaining protocol (i.e., the alternating-offers protocol) where there is uncertainty regarding one agent's reserve price. We provide an algorithm based on the combination of game theoretic analysis and search techniques which finds pure strategy sequential equilibria when they exist. Our approach is sound, complete and, in principle, can be applied to other uncertainty settings, e.g., uncertain discount factors, and uncertain weights of negotiation issues in multi-issue negotiation. We experimentally evaluate our algorithm with a number of case studies showing that the average computational time is less than $30 \mathrm{~s}$ and at least one pure strategy equilibrium exists in almost all (about 99.7\%) the bilateral bargaining scenarios we have looked at in the paper.
\end{abstract}

Keywords Negotiation · Bargaining · Autonomous agents $\cdot$ Equilibrium

This work was done while the author Bo An was a PhD student in the Department of Computer Science, University of Massachusetts, Amherst.

B. An $(\varangle)$

Department of Computer Science, University of Southern California, Los Angeles, CA, USA

e-mail: boa@usc.edu

N. Gatti

Dipartimento di Elettronica e Informazione, Politecnico di Milano, Milano, Italy

e-mail: ngatti@elet.polimi.it

V. Lesser

Department of Computer Science, University of Massachusetts, Amherst, MA, USA

e-mail: lesser@cs.umass.edu

Springer 


\section{Introduction}

Automated negotiation is an important research area bridging together economics, game theory, and artificial intelligence. It has received a prominent attention in recent years $[4,5,19,25]$ and its importance is widely acknowledged since intelligent agents that negotiate with each other on behalf of human users are expected to lead to more efficient negotiations [34]. A very common class of negotiation is bargaining. It refers to a situation in which individual agents have the possibility of concluding a mutually beneficial agreement which could not be imposed without all individuals' approval. We use the terms 'negotiation' and 'bargaining' interchangeably in this paper.

While there are many negotiation settings in electronic commerce transactions, the most common one (also the simplest one) is bilateral negotiation with a single negotiation issue. For instance, consider a scenario in which a buyer and a seller negotiate on the price of a good. In such a bargaining scenario, the two agents have different preferences over agreements. Thus agents need to make concessions toward a mutually acceptable agreement through a series of offers and counter offers. The negotiation fails if the two agents fail to achieve an agreement. Negotiations play a crucial role in many real-world scenarios, e.g., between a service provider and a customer to determine the price for providing a service.

The most widely studied bargaining protocol in strategic bargaining is the alternatingoffers protocol $[31,38]$. The alternating-offers protocol is considered to be the most satisfactory protocol of bargaining present in literature. Negotiation starts with one agent's making an offer to its opponent. After receiving an offer, an agent can either accept the most recent offer of its opponent or make a new counter offer, which implies that the negotiation process continues to the next round. The alternating-offers protocol captures the most important features of bargaining: bargaining consists of a sequence of offers and decisions to accept or reject these offers. The alternating-offers protocol has been widely used in the bargaining theory literature, e.g., $[4,5,19,32,35]$, just to name a few.

There are two main approaches for the study of bargaining, one formal and the other heuristic. The formal approach is based on game theory and aims at finding strategies that are in equilibrium (a brief survey follows). The difficulty of finding an equilibrium for problems that involve uncertainty, except for some special cases, has led researchers to develop heuristic approaches. According to this second approach, agents follow heuristic tactics that, even if producing non-equilibrium outcomes, find mutually satisfactory agreements. Well known examples are [5,14,27,36,37]. The two approaches have several interconnections, the former providing insights for the latter.

This paper focuses on the first (game theoretic) approach and, more specifically, on one of the most challenging open bargaining problems: finding agents' rational strategies in uncertain information bilateral bargaining with the alternating-offers protocol. Specifically, we consider one-sided uncertainty regarding the buyer's reserve price. That is, the buyer's reserve price is only known to the buyer and the seller only knows the probability distribution of the buyer's reserve price, which is common knowledge. ${ }^{1}$ All other information (e.g., the seller's reserve price, agents' discount factors, negotiation deadlines) is public. In addition, we assume that each agent has a negotiation deadline. The infinite horizon assumption, which is usually made in game theory literature (e.g., $[9,10,32])$, is not realistic in real-world applications [35]. This bargaining problem is customarily modeled as a Bayesian extensive-form game with infinite number of strategies since price is a continuous value. The

1 We also show in this paper that our approach can be applied to bargaining games with two-sided uncertainty and other cases. 
sequential equilibrium [18] is the appropriate solution concept for imperfect information extensive form games including our bargaining game. A sequential equilibrium specifies a pair: a system of beliefs that prescribes how agents' beliefs must be updated during the game and strategies that prescribe how agents should act. In a sequential equilibrium there is a circularity between the belief system and strategies: strategies must be sequentially rational given the belief system and belief system must be consistent with respect to strategies.

The study of the alternating-offers protocol with uncertain information is well known to be hard and there are still many open problems [19]. The microeconomics literature provides a number of closed form results with very narrow uncertainty settings. For instance, Rubinstein [32] considered bilateral infinite horizon bargaining with uncertainty over two possible discount factors. Gatti et al. [19] analyzed bilateral bargaining with one-sided uncertain deadlines. Chatterjee et al. $[9,10]$ studied bilateral infinite horizon bargaining with two-type uncertainty over the reservation values. The absence of agents' deadlines makes these last two results nonapplicable to the situation with deadlines. An et al. [3] only consider two-type uncertainty about reserve prices and their approach cannot be applied to the multiple type case. Operations research inspired equilibrium calculation algorithms (e.g., [29]) work only on games with finite number of strategies, and therefore cannot be applied to bargaining in which each agent's strategy space is continuous. Similarly, enumeration based methods [30] cannot be applied to continuous strategy space scenarios. One approach that is applicable to bargaining games with one-sided uncertainty is presented in [8]. The algorithm we propose in this paper outperforms the algorithm in [8] as follows. (1) It is much faster, allowing the solving of negotiations within a given deadline that the algorithm in [8] cannot solve (in addition, the algorithm in [8] suffers from memory space problems, while our algorithm does not): our algorithm solves settings with nine types and deadline 14 in a reasonable time, while the algorithm in [8] suffers from memory space problems even with five types. (2) It produces a pure strategy equilibrium that is in general more satisfactory than mixed strategy equilibrium (explained later). (3) It produces Pareto efficient equilibria, while the algorithm in [8] does not provide any guarantee and can find (unsatisfactory) Pareto dominated equilibria. (4) It finds all the equilibria and therefore our algorithm can be easily combined with an algorithm to select an equilibrium given a specific criterion. Ceppi et al. [8] only finds one equilibrium and thus additional criterion for selection can not be included in their approach.

Several attempts to extend the backward induction method [18] to bargaining games with uncertainty have been tried, but they work for very restrictive cases. This is because in the computation of the equilibrium they break down the circularity between strategies and the belief system. For example, Fatima etal. $[16,17]$ present a polynomial time algorithm to produce equilibrium strategies in multi-issue bargaining with uncertain reserve prices. By exploiting backward induction, their algorithm searches agents' strategy space from the deadline to the beginning of negotiation with the initial beliefs. Once the optimal strategies at the beginning of negotiation have been found, the system of beliefs are designed to be consistent with them. However, the optimization in their approach is myopic since it does not take into account the possibility that an agent may deviate by profitably exploiting the updated beliefs caused by the deviation. As a result, the strategies found by their approach are not guaranteed to be sequentially rational given the designed system of beliefs, as shown in [19] for the case with uncertain deadlines. We will further expand on this observation by showing that the strategies developed by Fatima et al. $[16,17]$ are not sequentially rational in cases where there is uncertainty over reserve prices.

Furthermore, few complexity results on the computation of a sequential equilibrium of a bargaining game are known. To the best of our knowledge, the only proven result is provided in [19], where the authors show that a mixed strategy equilibrium can be computed in 
polynomial time with one-sided uncertainty over the deadlines. However, with this kind of uncertainty the problem presents a very specific structure that makes it easy to be solved, but such structure is not present when uncertainty is over other parameters. Furthermore, with finite games, the problem of computing an equilibrium in mixed strategies is PPAD-hard [29] and in pure strategies is NP-hard [24] even with simple games (two actions per agent), but no general result is known when actions are continuous. A positive result on the sequential equilibrium is that it is possible to verify whether a strategy profile is a sequential equilibrium in polynomial time regardless of the number of agents [21].

The main contribution of this paper is the development of a novel algorithm to find all pure strategy sequential equilibria ${ }^{2}$ in bilateral bargaining with one-sided, multi-type uncertainty. Our algorithm combines together game theoretic analysis with state space search techniques and it is sound and complete. Our approach is based on the following two observations:

1. with pure strategies, the buyer's possible choices regarding whether different buyer types behave in the same way or in different ways at a decision making point are finite,

2. with pure strategies, the seller's possible beliefs regarding whether different buyer types will accept or reject its offer are finite.

We employ a backward approach to find sequential equilibria in the context of a forward search process: to compute agents' equilibrium strategy at a continuation game with certain belief, we search forward to find agents' equilibria strategies in its continuation game with different beliefs first by considering agents' all belief update rules as well as possible choices regarding whether different buyer types behave in the same way or in different ways; then we derive theoretically the agents' optimal strategies by applying backward induction and check equilibrium existence conditions. Our algorithm has a computational complexity that is exponential in the length of the bargaining, but our experimental evaluation shows that it solves bargaining games that are complex enough within a reasonable time. In addition, because there is potentially more than one equilibrium, we have designed our algorithm to find all of them. This allows one to select an equilibrium according to some criterion that depends on the application. As in all the previous work on strategic bargaining, we leave open the problem of how to define the criteria for the selection of the equilibrium.

We focus on pure strategy equilibria for the following reasons. The concept of mixed strategies is very useful for games having no pure strategy equilibrium. However, the concept of mixed strategies has been criticized for being "intuitively problematic" since randomization lacks behavioral support [6]. When mixed strategies are considered, the number of sequential equilibria of the game usually increases and coordination problems of choosing an equilibrium strategy profile has not been fully addressed. Fortunately, simulation results show that there is at least one pure strategy sequential equilibrium in $99.7 \%$ of various bilateral bargaining games in which the minimum deadline of the two agents is no higher than 14 and the number of buyer types is no more than 9. Additionally, in our experimental results we found that as the number of uncertain types and deadlines increase, all cases had at least one sequential equilibrium.

The rest of this paper is organized as follows. We start with a discussion of complete information bilateral negotiation in Sect. 2 which sets a context for Sect. 3 that introduces uncertainty into our bargaining game. Section 4 presents our approach for computing sequential equilibria. Section 5 shows how to compute the buyer's equilibrium offer and Sect. 6 shows how to compute the seller's equilibrium offer. Section 7 analyzes equilibrium existence. Section 8 discusses two potential applications of our approach. Section 9 discusses related work

${ }^{2}$ Strategies are pure when actions are played either with a probability of one or with a probability of zero. 
and gives an example to show how our approach leads to a different (and the correct) result from the approach designed by Fatima et al. $[16,17]$. Section 10 concludes this paper and outlines some future research directions.

\section{Bargaining with complete information}

This section describes the discrete time bargaining between a buyer $\mathbf{b}$ and a seller $\mathbf{s}$. We start with describing how to compute agents' equilibrium strategies in the complete information setting since this calculation will be used as part of the algorithm for the incomplete information setting. The bilateral bargaining game with complete information has been analyzed in [19] and here we follow the reasoning in [19]. The seller wants to sell a single indivisible good to the buyer for a price. All the agents enter the market at time 0 . An alternating-offers bargaining protocol is utilized. Formally, the buyer $\mathbf{b}$ and the seller $\mathbf{s}$ can act at times $t \in \mathbb{N}$. The player function $\iota: \mathbb{N} \rightarrow\{\mathbf{b}, \mathbf{s}\}$ returns the agent that acts at time $t$ and is such that $\iota(t) \neq \iota(t+1)$, i.e., a pair of agents bargain by making offers in alternate fashion. This paper focuses on single-issue negotiation but this model can be easily extended to handle multi-issue negotiation [13]. Table 1 lists the main symbols used in this paper.

Possible actions $\sigma_{l(t)}^{t}$ of agent $\iota(t)$ at any time point $t>0$ are:

1. offer $[x]$, where $x \in \mathbb{R}$ is the proposed price for the good;

2. exit, which indicates that negotiation fails;

3. accept, which indicates that $\mathbf{b}$ and $\mathbf{s}$ have reached an agreement.

At time point $t=0$, action accept is not allowed. If $\sigma_{t(t)}^{t}=$ accept the bargaining stops and the outcome is $o=(x, t)$, where $x$ is the value such that $\sigma_{t(t-1)}^{t-1}=\operatorname{offer}[x]$. This is to

Table 1 Used symbols

\begin{tabular}{ll}
\hline $\mathrm{b}$ & The buyer \\
$\mathrm{b}_{\mathrm{i}}$ & The type $i$ of the buyer \\
$\mathrm{s}$ & The seller \\
$\iota(t)$ & The agent acting at time $t$ \\
$\sigma_{l(t)}^{t}$ & The action of $\iota(t)$ at time $t$ \\
$\mathrm{RP}_{\mathrm{a}}$ & The reserve price of agent a \\
$T_{\mathrm{a}}$ & The deadline of agent a \\
$\delta_{\mathrm{a}}$ & The discount factor of agent a \\
$T$ & min( $\left.T_{\mathrm{b}}, T_{\mathrm{s}}\right)$ \\
$x^{*}(t)$ & The optimal offer of agent $\iota(t)$ at $t$ in complete information setting \\
$\mu(t) / \Delta_{\mathrm{b}}^{t}$ & The seller's belief at time $t$ \\
$\omega_{\mathrm{b}_{\mathrm{i}}}^{0}$ & The probability that $\mathrm{b}$ is of type $\mathrm{b}_{\mathrm{i}}$ at time 0 \\
$o_{\mathrm{b}_{\mathrm{i}}}$ & The bargaining outcome if $\mathrm{b}$ is of type $\mathrm{b}_{\mathrm{i}}$ \\
$\mathrm{b}_{h}\left(\Delta_{\mathrm{b}}^{t}\right)$ & The buyer type with the highest reserve price in buyer types $\Delta_{\mathrm{b}}^{t}$ \\
$e_{\mathrm{S}}^{t} \mid \mu(t)$ & The equivalent offer of $\mathrm{s}$ \\
$e_{\mathrm{b}_{\mathrm{i}}}^{t} \mid \mu(t)$ & The equivalent offer of buyer $\mathrm{b}_{i}$ \\
$E B O\left(\mathrm{~b}_{i}, x, t\right)$ & The equilibrium bargaining outcome of $\mathrm{b}_{i}$ if it offers $x$ at time $t$ \\
$E B O\left(\mathrm{~b}_{i}, \wp\right)$ & The equilibrium bargaining outcome of $\mathrm{b}_{i}$ if it follows $\wp$
\end{tabular}


say that the agents agree on the value $x$ at time point $t$. If $\sigma_{l(t)}^{t}=$ exit the bargaining stops and the outcome is $F A I L$. Otherwise the bargaining continues to the next time point.

Each agent $\mathbf{a} \in\{\mathbf{b}, \mathbf{s}\}$ has a utility function $U_{\mathbf{a}}:(\mathbb{R} \times \mathbb{N}) \cup\{F A I L\} \rightarrow \mathbb{R}$, which represents its gain over the possible bargaining outcomes. Each utility function $U_{\mathbf{a}}$ depends on agent a's reserve price $\mathrm{RP}_{\mathbf{a}} \in \mathbb{R}^{+}$, temporal discount factor $\delta_{\mathbf{a}} \in(0,1){ }^{3}$ and deadline $T_{\mathbf{a}} \in \mathbb{N}, T_{\mathbf{a}}>0$. If the bargaining outcome is $o=(x, t)$, then the utility function $U_{\mathbf{a}}$ for agent $\mathbf{a}$ is defined as:

$$
U_{\mathbf{a}}(x, t)= \begin{cases}\left(\mathrm{RP}_{\mathbf{a}}-x\right) \cdot\left(\delta_{\mathbf{a}}\right)^{t} & \text { if } t \leq T_{\mathbf{a}} \text { and } \mathbf{a} \text { is a buyer } \\ \left(x-\mathrm{RP}_{\mathbf{a}}\right) \cdot\left(\delta_{\mathbf{a}}\right)^{t} & \text { if } t \leq T_{\mathbf{a}} \text { and } \mathbf{a} \text { is a seller } \\ \epsilon<0 & \text { otherwise }\end{cases}
$$

If the negotiation outcome is $F A I L$, the utility for agent $\mathbf{a}$ is $U_{\mathbf{a}}(F A I L)=0$. Notice that the assignment of a strictly negative value $\epsilon<0$ to $U_{\mathbf{a}}$ after a's deadline allows one to capture the essence of the deadline: an agent, after its deadline, strictly prefers to exit the negotiation rather than to reach any agreement. Finally, we assume the feasibility of the problem, i.e., $\mathrm{RP}_{\mathbf{b}} \geq \mathrm{RP}_{\mathbf{s}}$.

With complete information the appropriate solution concept for the bargaining game is the subgame perfect equilibrium in which agents' strategies are in equilibrium in every possible subgame [18]. Note that there is no deadline constraint in the negotiation protocol, which indicates that agents are allowed to offer and counteroffer after their deadlines have expired. However, the deadline constraint is in both agents' utility functions such that no rational agent will continue negotiation after its deadline. Therefore, the bargaining game is a finite horizon game and the subgame perfect equilibrium can be found employing the backward induction method.

Initially, it is determined that the game rationally stops at time point $T=\min \left(T_{\mathbf{b}}, T_{\mathbf{S}}\right)$. The equilibrium outcome of every subgame starting from $t \geq T$ is $F A I L$, since at least one agent will exit from bargaining. Therefore, at $t=T$ agent $\iota(T)$ would accept any offer $x$ which gives it a utility not worse than $F A I L$, namely, any offer $x$ such that $U_{\iota(T)}(x, T) \geq 0$. From $t=T-1$ back to $t=0$ it is possible to find the optimal offer agent $\iota(t)$ can make at $t$, if it makes an offer, and the offers that it would accept. $x^{*}(t)$ denotes the optimal offer of agent $\iota(t)$ at $t . x^{*}(t)$ is the offer such that, if $t<T-1$, agent $\iota(t+1)$ is indifferent at $t+1$ between accepting it and rejecting it to make its optimal offer $x^{*}(t+1)$ and, if $t=T-1$, agent $\iota(t+1)$ is indifferent at $t+1$ between accepting it and exiting. Formally, $x^{*}(t)$ is such that $U_{l(t+1)}\left(x^{*}(t), t\right)=U_{\iota(t+1)}\left(x^{*}(t+1), t+1\right)$ if $t<T-1$ and $U_{l(t+1)}\left(x^{*}(t), t\right)=0$ if $t=T-1$. The offers agent $\iota(t)$ would accept at $t$ are all those offers that give it a utility no worse than the utility given by offering $x^{*}(t)$. The equilibrium strategy of any subgame starting from $0 \leq t<T$ prescribes that agent $\iota(t)$ offers $x^{*}(t)$ at $t$ and agent $\iota(t+1)$ accepts it at $t+1$.

Backward propagation is used to provide a recursive formula for $x^{*}(t)$ : given value $x$ for agent $\mathbf{a}$, we call the value $y$ the result of a one-step backward propagation of the value $x$ for agent a such that $U_{\mathbf{a}}(y, t-1)=U_{\mathbf{a}}(x, t)$; we employ the arrow notation $x_{\leftarrow \mathbf{a}}$ for backward propagations. Formally, $x_{\leftarrow \mathbf{b}}=\mathrm{RP}_{\mathbf{b}}-\left(\mathrm{RP}_{\mathbf{b}}-x\right) \cdot \delta_{\mathbf{b}}$ and $x_{\leftarrow \mathbf{s}}=\mathrm{RP}_{\mathbf{s}}+\left(x-\mathrm{RP}_{\mathbf{s}}\right) \cdot \delta_{\mathbf{s}}$. If a value $x$ is backward propagated $n$ times for agent a, we write $x_{\leftarrow n[\mathbf{a}]}$, e.g., $x_{\leftarrow 2[\mathbf{a}]}=\left(x_{\leftarrow \mathbf{a}}\right)_{\leftarrow \mathbf{a}}$. If a value is backward propagated for more than one agent, we list them left to right in the

3 A discount factor is used to model bargaining cost, which is a common assumption in the bargaining literature $[16,19,31,32]$. 


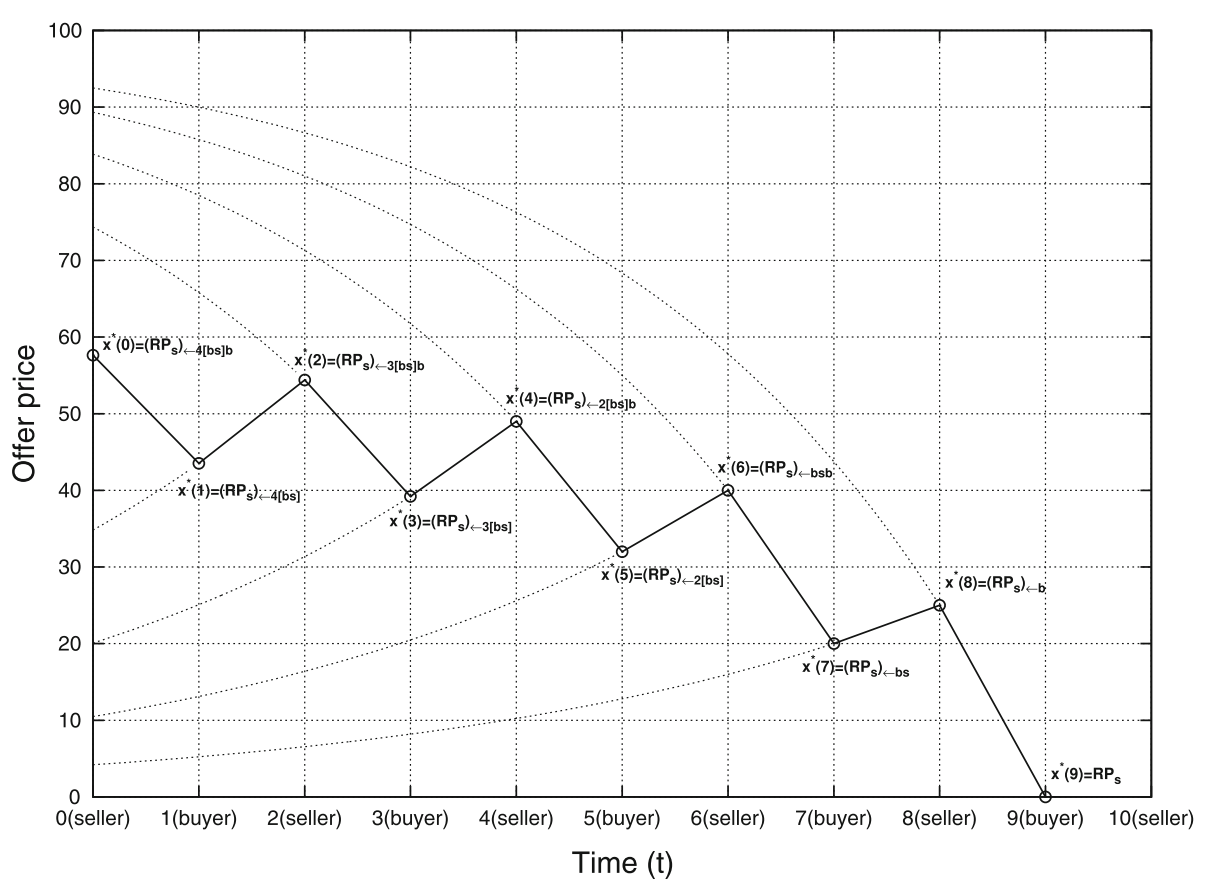

Fig. 1 Backward induction construction with $\mathrm{RP}_{\mathrm{b}}=100, \mathrm{RP}_{\mathrm{s}}=0, \iota(0)=\mathrm{s}, \delta_{\mathrm{b}}=0.75, \delta_{\mathrm{s}}=0.8, T_{\mathrm{b}}=10$, $T_{\mathrm{S}}=11$; at each time point $t$ the optimal offer $x^{*}(t)$ is marked; the dashed lines are isoutility curves

subscript, e.g., $x_{\leftarrow \mathbf{b} 2[\mathbf{s}]}=\left(\left(x_{\leftarrow \mathbf{b}}\right)_{\leftarrow \mathbf{s}}\right)_{\leftarrow \mathbf{s}}$. The values of $x^{*}(t)$ can be calculated recursively from $t=T-1$ back to $t=0$ as follows:

$$
x^{*}(t)= \begin{cases}\mathrm{RP}_{\iota(t+1)} & \text { if } t=T-1 \\ \left(x^{*}(t+1)\right)_{\leftarrow \iota(t+1)} & \text { if } t<T-1\end{cases}
$$

Theorem 1 The following inequalities hold: $x_{\leftarrow \mathbf{b}} \geq x$ and $x_{\leftarrow \mathbf{s}} \leq x$.

Proof We can easily prove this from the backward propagation process. We have $x_{\leftarrow \mathbf{b}} \geq x$ as $x \leftarrow \mathbf{b}-x=\mathrm{RP}_{\mathbf{b}}-\left(\mathrm{RP}_{\mathbf{b}}-x\right) \cdot \delta_{\mathbf{b}}-x=\left(1-\delta_{\mathbf{b}}\right)\left(\mathrm{RP}_{\mathbf{b}}-x\right) \geq 0$, which indicates $x \leftarrow \mathbf{b} \geq x$. Similarly, we have $x_{\leftarrow \mathbf{s}} \leq x$ as $x_{\leftarrow \mathbf{s}}-x=\mathrm{RP}_{\mathbf{s}}+\left(x-\mathrm{RP}_{\mathbf{s}}\right) \cdot \delta_{\mathbf{s}}-x=\left(\delta_{\mathbf{s}}-1\right)\left(x-\mathrm{RP}_{\mathbf{s}}\right) \leq 0$.

Figure 1 shows an example of backward induction construction with parameters $\mathrm{RP}_{\mathbf{b}}=$ $100, \mathrm{RP}_{\mathbf{s}}=0, \iota(0)=\mathbf{s}, \delta_{\mathbf{b}}=0.75, \delta_{\mathbf{s}}=0.8, T_{\mathbf{b}}=10$, and $T_{\mathbf{s}}=11$. The backward induction process starts from time $T=\min \left\{T_{\mathbf{b}}, T_{\mathbf{s}}\right\}=10$. At time 10 , the seller is willing to accept any offer which is no less than its reserve price and thus the optimal offer at time $t=9$ is $x^{*}(9)=\mathrm{RP}_{\mathrm{s}}=0$. The optimal offer of the seller at time $t=8$ is $x^{*}(8)=\left(\mathrm{RP}_{\mathbf{s}}\right)_{\leftarrow \mathbf{b}}=\mathrm{RP}_{\mathbf{b}}-\left(\mathrm{RP}_{\mathbf{b}}-\mathrm{RP}_{\mathbf{s}}\right) \cdot \delta_{\mathbf{b}}=25$. Analogously, the optimal offer of the buyer at time $t=7$ is $x^{*}(7)=\left(x^{*}(8)\right)_{\leftarrow \mathrm{s}}=\mathrm{RP}_{\mathbf{s}}+\left(x^{*}(8)-\mathrm{RP}_{\mathbf{s}}\right) \cdot \delta_{\mathbf{s}}=20$. Following this procedure, we can get agents' optimal offers from time $t=6$ to the initial time point $t=0$.

Finally, agents' equilibrium strategies can be defined on the basis of $x^{*}(t)$ as follows: 


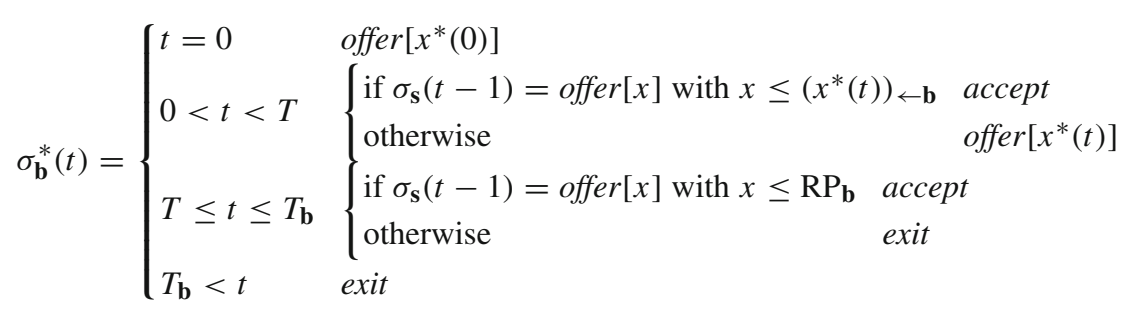

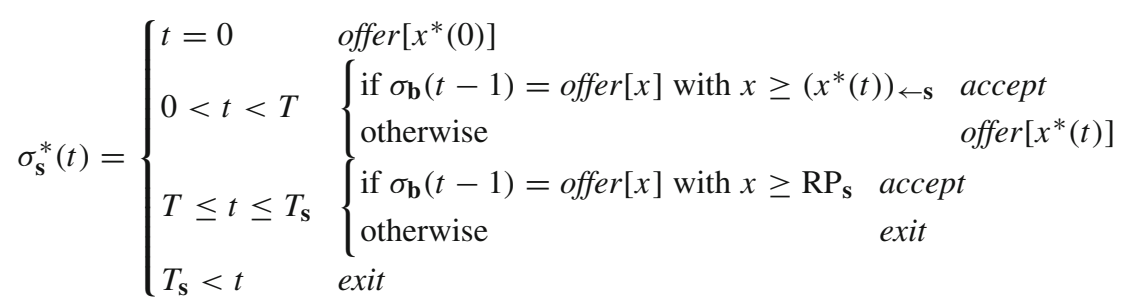

We can see that the above strategies constitute the unique subgame perfect equilibrium of bargaining with complete information. The equilibrium can be found in time linear in the maximum deadline of the two agents. At the equilibrium, the two agents reach an agreement at time $t=1$ and the agreement price is $x^{*}(0)$.

\section{One-sided uncertainty about reserve prices}

In this section, we modifies the complete information bargaining model in the previous section by introducing one-sided uncertainty regarding the buyer's reserve price.

In the presence of incomplete information, it is customary in game theory to introduce probability distributions over the parameters that are not known by the agents, which leads to games with uncertain information. By Harsanyi's transformation, uncertain information games are cast into imperfect information games where players can be of different types and there is uncertainty over players' type. The most widely used solution concept from game theory for an extensive-form bargaining game with imperfect information is sequential equilibrium [18]. A sequential equilibrium is a pair $a=\langle\mu, \sigma\rangle$ (also called an assessment) where $\mu$ is a belief system that specifies how agents' beliefs about the other agents' type evolve during the game and $\sigma$ specifies agents' strategies. At an equilibrium $\mu$ must be consistent with respect to $\sigma$ and $\sigma$ must be sequentially rational given $\mu$. Informally, the rationality requirement says that after every possible sequence of actions, an agent's strategy must maximize its expected utility given its beliefs and its opponent's equilibrium strategy. An assessment a is consistent (in the sense of Kreps [28]) if there exists a sequence of totally mixed strategy profiles (with associated sensible beliefs updated according to Bayes' rule) that converges to the equilibrium profile.

We assume the one-sided uncertainty regarding the type of the buyer $\mathbf{b}$ (the case of having uncertainty with the type of the seller $\mathbf{s}$ can be analyzed analogously). The buyer $\mathbf{b}$ can be of finitely many types $\left\{\mathbf{b}_{1}, \ldots, \mathbf{b}_{n}\right\}$ in which buyer type $\mathbf{b}_{i}$ has an associated reserve price $\mathrm{RP}_{i}$. The initial belief of $\mathbf{s}$ (i.e., s's belief at time 0 ) on $\mathbf{b}$ is described by $\mu(0)=\left\langle\Delta_{\mathbf{b}}^{0}, P_{\mathbf{b}}^{0}\right\rangle$ where $\Delta_{\mathbf{b}}^{0}=\left\{\mathbf{b}_{1}, \ldots, \mathbf{b}_{n}\right\}$ is the set of possible buyer's types and $P_{\mathbf{b}}^{0}=\left\{\omega_{\mathbf{b}_{1}}^{0}, \ldots, \omega_{\mathbf{b}_{n}}^{0}\right\}$, such that $\sum_{i} \omega_{\mathbf{b}_{i}}^{0}=1$, is the probability distribution over the buyer's types. The belief of $\mathbf{s}$ on the type of $\mathbf{b}$ at time $t$ is $\mu(t)=\left\langle\Delta_{\mathbf{b}}^{t}, P_{\mathbf{b}^{t}}^{t}\right\rangle$ where $\Delta_{\mathbf{b}}^{t} \subseteq \Delta_{\mathbf{b}}^{0}$ and $P_{\mathbf{b}}^{t}=\left\{\omega_{\mathbf{b}_{1}}^{t}, \ldots, \omega_{\mathbf{b}_{n}}^{t}\right\}$, with $\omega_{\mathbf{b}_{i}}^{t}$ 
denoting the probability assigned by $\mathbf{s}$ to $\mathbf{b}=\mathbf{b}_{i}$ at time $t$. Given an assessment $a=\langle\mu, \sigma\rangle$, there are multiple possible bargaining outcomes, one for each possible type of the buyer. We denote a type-specific outcome by $o_{\mathbf{b}_{i}}$ if $\mathbf{b}=\mathbf{b}_{i}$, while we denote a bargaining outcome as $o=\left\langle o_{\mathbf{b}_{1}}, \ldots, o_{\mathbf{b}_{n}}\right\rangle$.

Seller s's belief over the type of buyer $\mathbf{b}$ will evolve on the basis of the observed actions and the buyer's equilibrium strategies. As is customary in economic studies [32], we consider only stationary systems of beliefs, i.e., if $\mathbf{s}$ believes a b's type with zero probability at time point $t$, it will continue to believe such a type with zero probability at any time point $t^{\prime}>t$. We can therefore specify $\mu(t)$ by specifying $\Delta_{\mathbf{b}}^{t}$ and we use $\mu(t)$ and $\Delta_{\mathbf{b}}^{t}$ interchangeably in this paper. Moreover, given that $\mu(t)=\Delta_{\mathbf{b}}^{t}$ and we only consider pure strategies, the probability that $\mathbf{b}$ is of type $\mathbf{b}_{i} \in \Delta_{\mathbf{b}}^{t}$ is $\omega_{\mathbf{b}_{i}}\left(\Delta_{\mathbf{b}}^{t}\right)=\frac{\omega_{\mathbf{b}_{i}}^{0}}{\sum_{\mathbf{b}_{j} \in \Delta_{\mathbf{b}}^{t}} \omega_{\mathbf{b}_{j}}^{0}}$.

We need to also specify the belief system off the equilibrium path, i.e., when an agent takes an action that is not optimal. We use the optimistic conjectures $[32,33] .{ }^{4}$ That is, when buyer $\mathbf{b}$ acts off the equilibrium strategy, agent $\mathbf{s}$ will believe that agent $\mathbf{b}$ is of its "weakest" type, i.e., the type against which the seller would gain the most. This choice is made to assure the existence of the equilibrium for the largest subset of the space of the parameters [19]. If an equilibrium does not exist with optimistic conjectures then it does not exist. In our case, the weakest type is the buyer type with the highest reserve price (see Sect. 4.4 for the proof). That is, if $\mu(t-1)=\Delta_{\mathbf{b}}^{t-1}$ and $\mathbf{b}$ acts off the equilibrium strategy at time $t-1$, it follows that $\Delta_{\mathbf{b}}^{t}=\mathbf{b}_{h}\left(\Delta_{\mathbf{b}}^{t-1}\right)$ where $\mathbf{b}_{h}\left(\Delta_{\mathbf{b}}^{t-1}\right)$ is the buyer type with the highest reserve price in buyer types $\Delta_{\mathbf{b}}^{t-1}$.

We use the following simple real-life example to explain the applicability of our model. A buyer and a seller are negotiating for a used car utilizing the alternating-offers protocol. Each person has a reserve price. The seller's reserve price is the lowest price the seller can accept and such a reserve price could be the average contract price for the used car, which can be gained from trusted resources (e.g., Kelley Blue Book at http://www.kbb.com or Edmunds at http://www.edmunds.com) for used car prices. The buyer's reserve price represents the buyer's budget constraint, which is uncertain to the seller. For instance, the buyer's reserve price could be either low or high and the seller has a prior belief about the buyer's reserve price, e.g., the buyer has a low reserve price with $50 \%$. Such a prior belief is common knowledge for both players. The focus of this paper is computing both players' equilibrium strategies.

\section{The algorithm for finding all sequential equilibria}

This section first introduces the high level idea that motivates our approach. Following that we analyze some observations that can be used to reduce drastically the required computation based on our basic approach. Finally we introduce the algorithm for finding all sequential equilibria of a bilateral bargaining game with one-sided uncertainty.

\subsection{High level idea of the approach}

Our approach follows the spirit of backward induction: To compute agent a's equilibrium offer with belief $\Delta_{\mathbf{b}}$ at time $t<T-1$, agent a takes into account all the sequential equilibria

\footnotetext{
4 While this paper assumes optimistic conjectures, our approach can be used for any belief update rules for agents' actions off the equilibrium path. 
in the continuation game with different beliefs starting from time $t+1$. A continuation game is composed of an information set for one agent (buyer or seller) and all of its successor nodes from the original bilateral bargaining game. Note that there is no proper subgame for the bargaining game with uncertainty. There are continuation games starting from time points $0,1, \ldots$ Let $\Gamma(t)$ be the continuation game starting from time $t$. In the continuation game $\Gamma(t)$, agent $\iota(t)$ makes its offer at time $t$ first. Let $\Gamma\left(t, \Delta_{\mathbf{b}}\right)$ be the continuation game $\Gamma(t)$ with seller s's initial belief $\Delta_{\mathbf{b}}$. The problem of finding sequential equilibria for a bargaining problem is finding sequential equilibria for the continuation game $\Gamma\left(0, \Delta_{\mathbf{b}}^{0}\right)$ where $\Delta_{\mathbf{b}}^{0}$ is the seller's prior belief at time 0 .

The definition of a sequential equilibrium requires that after observing buyer b's counter offer at time $t$, seller $\mathbf{s}$ must update its belief about b's type using a belief update rule. If buyer $\mathbf{b}$ makes a new offer at time $t$, seller $\mathbf{s}$ observes two actions from buyer $\mathbf{b}$ :

1. Reject action: seller s's last offer is rejected by the buyer $\mathbf{b}$ if $t>0$. This is implicitly done when buyer makes a counteroffer, without making any 'reject' action. In some alternating-offers protocols studied in the literature (e.g., [16]), an agent is required to send a rejection message before making a new offer. However, these protocols are equivalent to ours. For the sake of simplicity, a rejecting agent does not need to send a rejection message in our protocol. Therefore, the rejection action still exists in our protocol in the case when an agent sends a counter-offer.

2. Offer action: Buyer $\mathbf{b}$ makes a new offer at time $t$.

Note that when buyer $\mathbf{b}$ makes an offer at time $t=0$, the seller only observes the offer action from the buyer since the seller has not made an offer yet.

Seller s will update its belief given all the actions of buyer $\mathbf{b}$. Therefore, there are two types of belief update rules:

1. Reject update rules applied when buyer b rejects seller s's offer;

2. Offer update rules applied when buyer $\mathbf{b}$ makes a new offer.

Definition 2 Assume seller s's belief before applying a reject update rule is $\Delta_{\mathbf{b}}$, a reject update rule is of the following form: If $x$ is rejected, s's belief about the type of buyer $\mathbf{b}$ is updated to $\Delta_{\mathbf{b}}^{\prime} \subseteq \Delta_{\mathbf{b}}$.

Definition 3 Assume seller s's belief before applying an offer update rule is $\Delta_{\mathbf{b}}$, an offer update rule has the following form: If buyer $\mathbf{b}$ offers $x$, seller $\mathbf{s}$ 's belief about the type of $\mathbf{b}$ is updated to $\Delta_{\mathbf{b}}^{\prime} \subseteq \Delta_{\mathbf{b}}$.

When buyer $\mathbf{b}$ makes an offer at time $t=0$, the seller will only apply its offer update rule. In any other situation (i.e., buyer b first rejects s's offer and then makes a new offer at time $t>0$ ), seller $\mathbf{s}$ will apply the reject update rule first and then apply the offer update rule. Thus, a reject update rule and an offer update rule consist of a belief update rule for the seller when it observes the buyer's offer at time $t>0$.

The definition of sequential equilibria requires that the seller's belief update rule should be consistent with the buyer's strategy in any sequential equilibrium. Assume that a reject update rule at time $t$ requires that the seller update its belief from $\Delta_{\mathbf{b}}$ to $\Delta_{\mathbf{b}}^{\prime}$ such that $\Delta_{\mathbf{b}}^{\prime} \subseteq \Delta_{\mathbf{b}}$. Bayesian consistency requires that all $\mathbf{b}_{i} \in \Delta_{\mathbf{b}}^{\prime}$ reject the seller's offer and all $\mathbf{b}_{j} \in \Delta_{\mathbf{b}}-\Delta_{\mathbf{b}}^{\prime}$ accept the offer. By the requirement of sequential rationality, we need to verify that it is each $\mathbf{b}_{i} \in \Delta_{\mathbf{b}}^{\prime}$ 's optimal strategy to reject the seller's offer and it is $\mathbf{b}_{j} \in \Delta_{\mathbf{b}}-\Delta_{\mathbf{b}}^{\prime}$ 's optimal strategy to accept the seller's offer. Similarly, an offer update rule also adds constraints to different buyer types' offering prices due to requirements of Bayesian consistency and sequential rationality. 


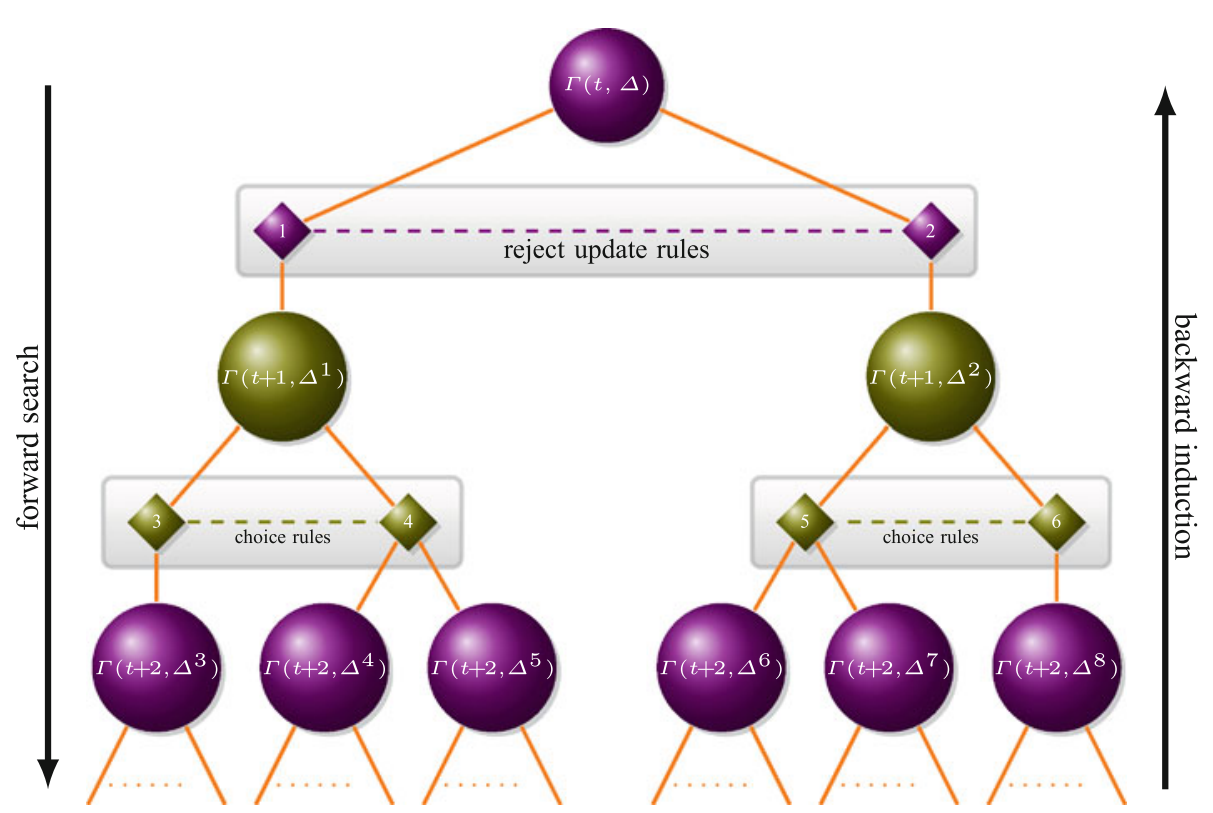

Fig. 2 A high level illustration of our approach $\left(\iota(t)=\mathbf{s}\right.$ and $\left.\left|\Delta^{0}\right|>1\right)$

While the seller is making an offer at time $t$ given the sequential equilibria for the continuation game $\Gamma(t+1)$ with different beliefs, the seller will consider different reject update rules and compute its equilibrium offer for each rule. With pure strategies, the seller's reject update rules are finite. The other situation is deciding the buyer's equilibrium offer at time $t$ given the sequential equilibria for the continuation game $\Gamma(t+1)$ with different beliefs, the buyer will consider different choice rules regarding whether different buyer types behave in the same way or behave in different ways. With pure strategies, buyer types' choice rules are finite. For each choice rule, we compute each buyer type's optimal offer and its corresponding offer update rule. While computing agents' equilibrium strategies, we also construct equilibrium existence conditions and check whether those conditions are satisfied.

Roughly, the idea of our approach is the following (see Fig. 2). To compute agents' equilibrium offers at a continuation game, we first compute sequential equilibria in its continuation game with different beliefs. Then we compute agents' equilibrium offers together with agents' belief update rules. There are two cases. While computing the seller's equilibrium strategy, we enumerate all possible reject update rules (e.g., reject update rules 1 and 2 in Fig. 2) and for each reject update rule, we first compute the seller's optimal strategy in the corresponding continuation game. For example, for the reject update rule 1 in Fig. 2, we first solve the continuation game $\Gamma\left(t+1, \Delta^{1}\right)$ where $\Delta^{1} \subseteq \Delta^{0}$ is the seller's updated belief if the seller's offer is rejected. While computing the buyer's equilibrium strategy, we consider all choice rules and compute different buyer types' optimal offer for each choice rule. For instance, for the choice rule 3 in Fig. 2, we need to first solve the continuation game $\Gamma\left(t+2, \Delta^{3}\right)$. There are two processes involved in computing all sequential equilibria: a forward search process to determine the set of continuation games to solve and a backward induction process to compute agents' equilibrium strategies based on all sequential equilibria of continuation games. Furthermore, we introduce some equilibrium existence conditions by 
considering the requirements of Bayesian consistency and sequential rationality: if they are satisfied, there is a sequential equilibrium in the continuation game.

Consider a bargaining problem with 2 buyer types $\left\{\mathbf{b}_{1}, \mathbf{b}_{2}\right\}$ and $T=5$. Our objective is to compute all sequential equilibria for the continuation game $\Gamma\left(0,\left\{\mathbf{b}_{1}, \mathbf{b}_{2}\right\}\right)$. Since $\iota(0)=\mathbf{s}$, we need to consider different reject update rules. Consider the reject update rule that the seller is making an offer $x$ that will only be accepted by buyer type $\mathbf{b}_{1}$, i.e., if the buyer rejects offer $x$, the seller will update its belief to $\left\{\mathbf{b}_{2}\right\}$. To compute the optimal offer $x$ at time $t=0$, we first compute all sequential equilibria for the continuation game $\Gamma\left(1,\left\{\mathbf{b}_{2}\right\}\right)$ starting from time $t=1$. For another reject update rule that the seller is making an offer $x$ that will be rejected by both buyer types, we need to first compute sequential equilibria for the continuation game $\Gamma\left(1,\left\{\mathbf{b}_{1}, \mathbf{b}_{2}\right\}\right)$ with the original belief. To compute sequential equilibria for the continuation game $\Gamma\left(1,\left\{\mathbf{b}_{1}, \mathbf{b}_{2}\right\}\right)$, we need to consider buyer types' different choice rules. Consider the choice rule that buyer type $\mathbf{b}_{1}$ makes an acceptable offer but buyer type $\mathbf{b}_{2}$ makes an offer that will be rejected. For this choice rule, we need to first compute sequential equilibria for continuation games $\Gamma\left(2,\left\{\mathbf{b}_{1}\right\}\right)$ and $\Gamma\left(2,\left\{\mathbf{b}_{2}\right\}\right)$ starting from time $t=2$. In the same way, we can recursively try different choice rules and reject update rules to compute all sequential equilibria of the bargaining game.

\subsection{Computation reduction}

This section provides some theoretical results which drastically reduce the computation complexity. Before we proceed, we introduce the concept of equivalent offer. In complete information bargaining, seller s's optimal offer $x^{*}(t)$ at time $t$ is the value to be propagated backward at time point $t-1$. That is, if $\mathbf{b}$ offers $\left(x^{*}(t)\right)_{\leftarrow \mathbf{s}}$ at time $t-1$, $\mathbf{s}$ will accept it at time $t$. With incomplete information, this property no longer holds since $\mathbf{s}$ will accept an offer if and only if the utility of accepting the offer is not less than the expected utility of making its optimal offer at time $t$. Given the equilibrium assessment $\left\langle\mu, \sigma^{*}\right\rangle$, the equilibrium expected utility of seller s's offer $x$ at time $t$, denoted as $E U_{\mathbf{s}}(x, t)$, is the expected utility of the seller's offering $x$ if (1) the seller's belief at time $t$ is $\mu(t)$ and (2) agents act according to the equilibrium strategies $\sigma^{*}$ from time $t$ on. The equivalent offer of s's offering $x$, denoted as $e_{\mathbf{s}}^{t} \mid \mu(t)$, is a value satisfying $U_{\mathbf{s}}\left(e_{\mathbf{s}}^{t} \mid \mu(t), t+1\right)=E U_{\mathbf{s}}(x, t) . e_{\mathbf{s}}^{t} \mid \mu(t)$ is the value to be propagated backward at time point $t-1$.

Similarly, the equivalent offer of buyer $\mathbf{b}_{i}$ 's offering $x$ at time $t$, denoted as $e_{\mathbf{b}_{i}}^{t} \mid \mu(t)$, is a value satisfying $U_{\mathbf{b}_{i}}\left(e_{\mathbf{b}_{i}}^{t} \mid \mu(t), t+1\right)=U_{\mathbf{b}_{i}}\left(E B O\left(\mathbf{b}_{i}, x, t\right)\right)$ where $E B O\left(\mathbf{b}_{i}, x, t\right)$ is the equilibrium bargaining outcome of $\mathbf{b}_{i}$ if it offers $x$ at time $t$. In addition, let $E B O\left(\mathbf{b}_{i}, \wp\right)$ denote the equilibrium bargaining outcome of $\mathbf{b}_{i}$ if agents follow the strategies specified by a sequential equilibrium $\wp$. Given a bargaining outcome $o c$, buyer $\mathbf{b}_{i}$ 's equivalent offer at time $t$ is given by function $\rho\left(\mathbf{b}_{i}, t, o c\right)$ which satisfies $U_{\mathbf{b}_{i}}\left(\rho\left(\mathbf{b}_{i}, t, o c\right), t+1\right)=U_{\mathbf{b}_{i}}(o c)$.

In an equilibrium, it is possible that the seller will make an offer that will be rejected by all the buyer types. Without loss of generality, we assume $\varpi$ be seller's offer that will be rejected by all buyer types. Assume that the seller's belief is $\Delta_{\mathbf{b}}$. A reject update rule specifies the seller's updated belief $\Delta_{\mathbf{b}}^{\prime} \subseteq \Delta_{\mathbf{b}}$ if the seller's offer is rejected. Therefore, the number of reject update rules are finite since the number of belief set $\Delta_{\mathbf{b}}^{\prime} \subseteq \Delta_{\mathbf{b}}$ is no more than $2^{\left|\Delta_{\mathbf{b}}\right|}$. However, the following theorem shows that there is no sequential equilibrium for most of the reject update rules.

Theorem 4 If there is a reject update rule with updated belief $\Delta_{\mathbf{b}}^{\prime} \subseteq \Delta_{\mathbf{b}}$ such that $\mathrm{RP}_{i}<\mathrm{RP}_{j}$ for buyer type $\mathbf{b}_{i} \in \Delta_{\mathbf{b}} \backslash \Delta_{\mathbf{b}}^{\prime}$ and buyer type $\mathbf{b}_{j} \in \Delta_{\mathbf{b}}^{\prime}$, agents' strategies are not sequentially rational. 
Proof This result can be proved by contradiction. If there is a sequential equilibrium with this reject update rule in which s's equilibrium offer at time $t$ is $x$, the following two conditions are satisfied:

1. $\mathbf{b}_{i}$ has no incentive to behave as $\mathbf{b}_{j}$, i.e., $U_{\mathbf{b}_{i}}(x, t+1) \geq U_{\mathbf{b}_{i}}\left(e_{\mathbf{b}_{j}}^{t+1} \mid \Delta_{\mathbf{b}}^{\prime}, t+2\right)$ where $e_{\mathbf{b}_{j}}^{t+1} \mid \Delta_{\mathbf{b}}^{\prime}$ is $\mathbf{b}_{j}$ 's equivalent offer in the continuation game starting from $t+1$ with belief $\Delta_{\mathbf{b}}^{\prime}$

2. $\mathbf{b}_{j}$ has no incentive to behave as $\mathbf{b}_{i}$, i.e., $U_{\mathbf{b}_{j}}\left(e_{\mathbf{b}_{j}}^{t+1} \mid \Delta_{\mathbf{b}}^{\prime}, t+2\right) \geq U_{\mathbf{b}_{j}}(x, t+1)$.

Condition (1) suggests that $x \leq\left(e_{\mathbf{b}_{j}}^{t+1} \mid \Delta_{\mathbf{b}}^{\prime}\right) \leftarrow \mathbf{b}_{i}$ and condition (2) indicates that $x \geq$ $\left(e_{\mathbf{b}_{j}}^{t+1} \mid \Delta_{\mathbf{b}}^{\prime}\right)_{\leftarrow} \mathbf{b}_{j}$. Therefore, equilibrium existence conditions requires that $\left(e_{\mathbf{b}_{j}}^{t+1} \mid \Delta_{\mathbf{b}}^{\prime}\right)_{\leftarrow} \mathbf{b}_{j} \leq$ $\left(e_{\mathbf{b}_{j}}^{t+1} \mid \Delta_{\mathbf{b}}^{\prime}\right)_{\leftarrow \mathbf{b}_{i}}$, which cannot be true since $\mathrm{RP}_{i}<\mathrm{RP}_{j}$.

Due to Theorem 4, we only need to consider reject update rules in which buyer types with higher reserve prices accept the seller's equilibrium offer while buyer types with lower reserve prices reject the seller's equilibrium offer. Those reject update rules are called feasible reject update rules.

Definition 5 If a reject update rule with updated belief $\Delta_{\mathbf{b}}^{\prime} \subseteq \Delta_{\mathbf{b}}$ satisfies the condition that $\mathrm{RP}_{i}>\mathrm{RP}_{j}$ for any buyer type $\mathbf{b}_{i} \in \Delta_{\mathbf{b}} \backslash \Delta_{\mathbf{b}}^{\prime}$ and any buyer type $\mathbf{b}_{j} \in \Delta_{\mathbf{b}}^{\prime}$, it is a feasible reject update rule.

Assume that the seller's belief before applying a reject update rule is $\Delta_{\mathbf{b}}$. The total number of feasible reject update rules we need to consider is at most $\left|\Delta_{\mathbf{b}}\right|$ rather than $2^{\left|\Delta_{\mathbf{b}}\right|}$, which are the total number of reject update rules. For each feasible reject update rule at time $t$, we need to first compute the sequential equilibrium for the continuation game $\Gamma(t+1)$ with the corresponding updated belief if the seller's offer is rejected, i.e., $\Delta_{\mathbf{b}}^{\prime}$. Accordingly, we need to compute sequential equilibria for the continuation game with at most $\left|\Delta_{\mathbf{b}}\right|$ different beliefs.

In addition to the above rejected update rules in which according to the equilibrium strategy at least one buyer type will reject the seller's offer, we also need to consider the case that according to the equilibrium strategy, the seller's offer will be accepted by all buyer types. If the offer is rejected (i.e., the buyer is acting off the equilibrium path), the seller will update its belief to the buyer type with the highest reserve price according to the optimistic conjectures. We call this reject update rule as null reject update rule.

The other situation is deciding the buyer's equilibrium offer at time $t$. We use the term "choice rule" to characterize buyer types' strategies regarding whether they behave in the same way at a specific decision making point. With pure strategies, buyer types' choice rules are finite. Consider that the belief of $\mathbf{s}$ on the type of $\mathbf{b}$ at time $t$ is $\mu(t)=\Delta_{\mathbf{b}}$ where $\left|\Delta_{\mathbf{b}}\right|>1$ (note that if $\left|\Delta_{\mathbf{b}}\right|=1$, the bargaining from time $t$ becomes the trivial complete information bargaining) and $\iota(t)=\mathbf{b}$. Let the equilibrium offer of buyer type $\mathbf{b}_{i} \in \Delta_{\mathbf{b}}$ be $x_{\mathbf{b}_{i}}(t)$. After receiving b's offer, $\mathbf{s}$ will update its belief and decide whether to accept the offer from $\mathbf{b}$. Without loss of generality, we assume that $x_{\mathbf{b}_{i}}(t)=-1$ if $\mathbf{b}_{i}$ 's equilibrium offer will be rejected by seller $\mathbf{s}$ at time $t+1$. There are two situations: (1) All buyer types make the same offer. In this case, a pooling choice rule is chosen by different buyer types. (2) Buyer types make different offers. That is, a separating choice rule is used by different buyer types.

It is easy to see that there are two pooling choice rules depending on whether the seller will accept the offer at time $t+1$ in equilibrium: (1) accepting pooling choice rule in which all buyer types make the same acceptable offer to seller $\mathbf{s}$; (2) rejecting pooling choice rule in which all buyer types make the same rejectable offer (i.e., -1$)$ to seller $\mathbf{s}$. While the buyer 
adopts the separating choice rule, some buyer types' equilibrium offers are acceptable to the seller and the number of separating choice rules is drastically reduced due to the following theorem.

Theorem 6 There is no equilibrium assessment in pure strategies if buyer types make different acceptable offers at $t$.

Proof We can easily prove this by contradiction. Assume that there is a sequential equilibrium for a belief system in which at time $t$ such that $\iota(t)=\mathbf{b}$, buyer $\mathbf{b}_{i}$ makes an acceptable offer $x$ to $\mathbf{s}$ and buyer types $\mathbf{b}_{j}$ makes an acceptable offer $y$ to $\mathbf{s}$ such that $x \neq y$. If $x>y$, buyer $\mathbf{b}_{i}$ has an incentive to behave like buyer $\mathbf{b}_{j}$ by offering price $y$. The other direction is analogous.

Therefore, we only need to consider the following separating choice rules: buyer types $\Delta_{\mathbf{b}}^{a}$ make an acceptable offer to $\mathbf{s}$ at time $t$ but buyer types $\Delta_{\mathbf{b}}^{r}=\Delta_{\mathbf{b}} \backslash \Delta_{\mathbf{b}}^{a}$ make an offer (i.e., -1 ) that will be rejected by $\mathbf{s}$ at time $t$. The total number of partitions satisfying the condition $\Delta_{\mathbf{b}}^{a} \cup \Delta_{\mathbf{b}}^{r}=\Delta_{\mathbf{b}}$ is $2^{\left|\Delta_{\mathbf{b}}\right|}-2$. However, the following theorem indicates that we only need to consider at most $\left|\Delta_{\mathbf{b}}\right|$ different choice rules.

Theorem 7 Assume that $\mathbf{b}$ behaves in different ways at a continuation game with belief set $\Delta_{\mathbf{b}}$ where $\Delta_{\mathbf{b}}=\Delta_{\mathbf{b}}^{a} \cup \Delta_{\mathbf{b}}^{r}$ at time $t$. If there is a buyer type $\mathbf{b}_{i} \in \Delta_{\mathbf{b}}^{a}$ and a buyer $\mathbf{b}_{j} \in \Delta_{\mathbf{b}}^{r}$ such that $\mathrm{RP}_{i}<\mathrm{RP}_{j}$, there is no sequential equilibrium for this choice rule.

Proof This result can be proved by contradiction. If there is a sequential equilibrium, the following two conditions are satisfied: (1) Buyer type $\mathbf{b}_{i}$ has no incentive to behave as $\mathbf{b}_{j}$, i.e., $\left(e_{\mathbf{s}}^{t+1} \mid \Delta_{\mathbf{b}}^{a}\right)_{\leftarrow \mathbf{s}} \leq \rho\left(\mathbf{b}_{i}, t, E B O\left(\mathbf{b}_{j}, \wp^{r}\right)\right)$; and (2) Buyer type $\mathbf{b}_{j}$ has no incentive to behave as $\mathbf{b}_{i}$, i.e., $\rho\left(\mathbf{b}_{j}, t, E B O\left(\mathbf{b}_{j}, \wp^{r}\right)\right) \leq\left(e_{\mathbf{s}}^{t+1} \mid \Delta_{\mathbf{b}}^{a}\right)_{\leftarrow \mathbf{s}}$. Therefore, equilibrium existence requires that $\rho\left(\mathbf{b}_{j}, t, E B O\left(\mathbf{b}_{j}, \wp^{r}\right)\right) \leq \rho\left(\mathbf{b}_{i}, t, E B O\left(\mathbf{b}_{j}, \wp^{r}\right)\right)$.

Assume that $E B O\left(\mathbf{b}_{j}, \wp^{r}\right)=\left(x, t^{\prime}\right)$ where $T \geq t^{\prime}>t$. From the definition of equivalent offers, we have $\left(\mathrm{RP}_{j}-\rho\left(\mathbf{b}_{j}, t, E B O\left(\mathbf{b}_{j}, \wp^{r}\right)\right)\right) \cdot \delta_{\mathbf{b}}^{t+1}=\left(\mathrm{RP}_{j}-x\right) \cdot \delta_{\mathbf{b}}^{t^{\prime}}$, which can be rewritten as $\rho\left(\mathbf{b}_{j}, t, E B O\left(\mathbf{b}_{j}, \wp^{r}\right)\right)=\mathrm{RP}_{j}-\left(\mathrm{RP}_{j}-x\right) \cdot \delta_{\mathbf{b}}^{t^{\prime}-t-1}$. Similarly, we have $\rho\left(\mathbf{b}_{i}, t, E B O\left(\mathbf{b}_{j}, \wp^{r}\right)\right)=\mathrm{RP}_{i}-\left(\mathrm{RP}_{i}-x\right) \cdot \delta_{\mathbf{b}}^{t^{\prime}-t-1}$. Since $\mathrm{RP}_{i}<\mathrm{RP}_{j}$, it follows that $\rho\left(\mathbf{b}_{i}, t, E B O\left(\mathbf{b}_{j}, \wp^{r}\right)\right)<\rho\left(\mathbf{b}_{j}, t, E B O\left(\mathbf{b}_{j}, \wp^{r}\right)\right)$ which contradicts with equilibrium existence conditions.

Theorem 7 says that we only need to consider separating choice rules in which buyer types with higher reserve prices make an acceptable offer while buyer types with lower reserve prices make an offer that will be rejected by the seller. Those separating choice rules are called feasible separating choice rules:

Definition 8 A separating choice rule $\Delta_{\mathbf{b}}=\Delta_{\mathbf{b}}^{a} \cup \Delta_{\mathbf{b}}^{r}$ is a feasible separating choice rule if $\mathrm{RP}_{i}>\mathrm{RP}_{j}$ for any buyer type $\mathbf{b}_{i} \in \Delta_{\mathbf{b}}^{a}$ and any buyer type $\mathbf{b}_{j} \in \Delta_{\mathbf{b}}^{r}$.

Theorem 7 drastically reduces the number of separating choice rules we need to consider. Consider a belief set $\Delta_{\mathbf{b}}$ at time $t<T-1$ such that $\left|\Delta_{\mathbf{b}}\right|>1$ and $\iota(t)=\mathbf{b}$. The total number of partitions satisfying the condition $\Delta_{\mathbf{b}}^{a} \cup \Delta_{\mathbf{b}}^{r}=\Delta_{\mathbf{b}}$ is $2^{\left|\Delta_{\mathbf{b}}\right|}-2$. However, the total number of feasible separating choice rules is $\left|\Delta_{\mathbf{b}}\right|-1$. For each feasible choice rule at time $t$, we need to first compute the sequential equilibria for the continuation game $\Gamma(t+1)$ with beliefs $\Delta_{\mathbf{b}}^{a}$ and $\Delta_{\mathbf{b}}^{s}$. Accordingly, we need to compute sequential equilibria for the continuation game with at most $2\left(\left|\Delta_{\mathbf{b}}\right|-1\right)$ different beliefs.

We call the set of feasible separating choice rules together with the two pooling choice rules as feasible choice rules. Assume that the seller's belief before applying an offer update rule is $\Delta_{\mathbf{b}}$, there are $\left|\Delta_{\mathbf{b}}\right|+1$ feasible choice rules in total. 


\subsection{Outline of the algorithm}

Algorithms 1 and 2 outline the main steps for computing agents' equilibrium strategies in a continuation game based on the sequential equilibria in its continuation game with different beliefs. To compute a buyer agent's equilibrium offer, the buyer considers different feasible choice rules and for each choice rule, we need to consider all the sequential equilibria of the continuation game with beliefs corresponding to the choice rule since there may be multiple sequential equilibria for the continuation game with a specific belief. Different buyer types' equilibrium strategies are derived using backward induction (see Sect. 5). To compute the seller's equilibrium offer at a time point, we consider all the feasible reject update rules and for each reject update rule, we compute the sequential equilibria of the continuation game with the belief corresponding to the reject update rule. We compute the seller's equilibrium offer for each sequential equilibrium corresponding to a reject update rule and check equilibrium existence conditions (see Sect. 6).
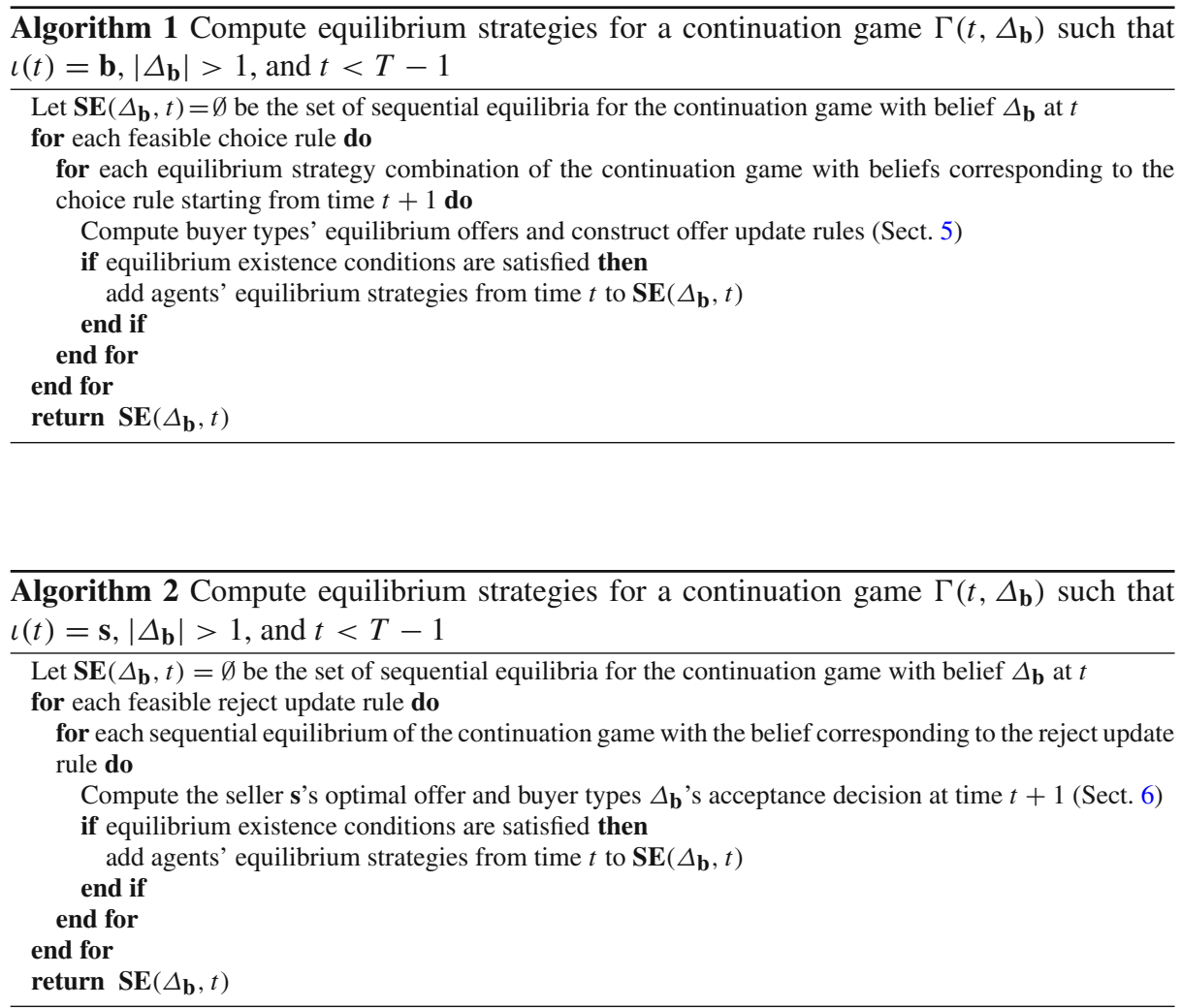

\subsection{Off the equilibrium path optimal strategies}

Before analyzing equilibrium strategies, we provide the optimal strategies in the situations seller $\mathbf{s}$ believes the buyer to be of one single type. There are two cases: (1) Seller $\mathbf{s}$ has the right belief about the type of the buyer $\mathbf{b}$. In this case, agents' equilibrium strategies are the 
equilibrium strategies of the corresponding complete information bargaining discussed in Sect. 2. Let $x_{\mathbf{b}_{i}}^{*}(t)$ be any agent optimal offer at time $t$ when $\mathbf{b}$ is of type $\mathbf{b}_{i}$ in this case. (2) Seller $\mathbf{s}$ has the wrong belief about the type of the buyer $\mathbf{b}$, i.e., $\mathbf{b}_{i}$ is believed to be $\mathbf{b}_{j}$.

Lemma $9 x_{\mathbf{b}_{i}}^{*}(t) \geq x_{\mathbf{b}_{j}}^{*}(t)$ if $\mathrm{RP}_{i}>\mathrm{RP}_{j}$.

Proof Case $1(\iota(T)=\mathbf{s})$. It follows that $x_{\mathbf{b}_{i}}^{*}(T-1)=x_{\mathbf{b}_{j}}^{*}(T-1)=\mathrm{RP}_{\mathbf{s}}$. Then $x_{\mathbf{b}_{i}}^{*}(T-2)=$ $\mathrm{RP}_{i}\left(1-\delta_{\mathbf{b}}\right)+\delta_{\mathbf{b}} x_{\mathbf{b}_{i}}^{*}(T-1)>x_{\mathbf{b}_{j}}^{*}(T-2)=\mathrm{RP}_{j}\left(1-\delta_{\mathbf{b}}\right)+\delta_{\mathbf{b}} x_{\mathbf{b}_{i}}^{*}(T-1)$. Similarly, we have $x_{\mathbf{b}_{i}}^{*}(T-3)=\operatorname{RP}_{\mathbf{s}}\left(1-\delta_{\mathbf{s}}\right)+\delta_{\mathbf{s}} x_{\mathbf{b}_{i}}^{*}(T-2)$ and $x_{\mathbf{b}_{j}}^{*}(T-3)=\operatorname{RP}_{\mathbf{s}}\left(1-\delta_{\mathbf{s}}\right)+\delta_{\mathbf{s}} x_{\mathbf{b}_{j}}^{*}(T-2)$. Thus we have $x_{\mathbf{b}_{i}}^{*}(T-3)>x_{\mathbf{b}_{j}}^{*}(T-3)$. Recursively, we have $x_{\mathbf{b}_{i}}^{*}(t)>x_{\mathbf{b}_{j}}^{*}(t)$ for $t<T-3$.

Case $2(\iota(T)=\mathbf{b})$. It follows that $x_{\mathbf{b}_{i}}^{*}(T-1)=\mathrm{RP}_{i}>x_{\mathbf{b}_{j}}^{*}(T-1)=\mathrm{RP}_{j}$. Then at time $T-2$, we have $x_{\mathbf{b}_{i}}^{*}(T-2)=\operatorname{RP}_{\mathbf{s}}\left(1-\delta_{\mathbf{s}}\right)+\delta_{\mathbf{s}} x_{\mathbf{b}_{i}}^{*}(T-1)$ and $x_{\mathbf{b}_{j}}^{*}(T-2)=\operatorname{RP}_{\mathbf{s}}\left(1-\delta_{\mathbf{s}}\right)+$ $\delta_{\mathbf{s}} x_{\mathbf{b}_{j}}^{*}(T-1)$. Thus, $x_{\mathbf{b}_{i}}^{*}(T-2)>x_{\mathbf{b}_{j}}^{*}(T-2)$. Recursively, we have $x_{\mathbf{b}_{i}}^{*}(t)>x_{\mathbf{b}_{j}}^{*}(t)$ for $t<T-2$.

We can see that $\mathbf{b}_{i}$ is weaker than $\mathbf{b}_{j}$ in terms of its offering price at each time point in complete information bargaining. Similarly, we can get $\mathrm{RP}_{i}-x_{\mathbf{b}_{i}}^{*}(t) \geq \mathrm{RP}_{j}-x_{\mathbf{b}_{j}}^{*}(t)$. $\mathrm{RP}_{i}-x_{\mathbf{b}_{i}}^{*}(0)$ is the gain (utility) of $\mathbf{b}_{i}$ in complete information bargaining and $\mathrm{RP}_{j}-x_{\mathbf{b}_{j}}^{*}(0)$ is the gain (utility) of $\mathbf{b}_{j}$ in complete information bargaining.

Lemma $10 x_{\mathbf{b}_{i}}^{*}(t) \leq\left(x_{\mathbf{b}_{i}}^{*}(t+1)\right)_{\leftarrow \mathbf{b}_{i}}$ and $x_{\mathbf{b}_{j}}^{*}(t) \leq\left(x_{\mathbf{b}_{j}}^{*}(t+1)\right) \leftarrow \mathbf{b}_{j}$ if $\operatorname{RP}_{i}>\operatorname{RP}_{j}$.

Proof We can get this result by following the same procedure in the proof of Lemma 9.

Lemma 10 indicates that the buyer will accept sellers' lowest equilibrium price in complete information bargaining, i.e., agents will reach a final agreement at time $T-2$ in the complete information bargaining case.

Agents' equilibrium strategies when seller $\mathbf{s}$ has the wrong belief about the type of the buyer $\mathbf{b}$ are specified in the following theorem.

Theorem 11 If seller $\mathbf{s}$ has the wrong belief about the type of $\mathbf{b}$, its optimal strategies are those in complete information bargaining. Assume that $\mathrm{RP}_{i}>\mathrm{RP}_{j}$. The optimal strategies $\sigma_{\mathbf{b}_{i}}^{*}(t) \mid\left\{\mathbf{b}_{j}\right\}$ of buyer $\mathbf{b}_{i}$ when it is believed to be $\mathbf{b}_{j}$ are:

$$
\sigma_{\mathbf{b}_{i}}^{*}(t) \mid\left\{\mathbf{b}_{j}\right\}= \begin{cases}\text { accepty } & \text { if } y \leq\left(x_{\mathbf{b}_{j}}^{*}(t)\right)_{\leftarrow} \mathbf{b}_{i} \\ \text { offer } x_{\mathbf{b}_{j}}^{*}(t) & \text { otherwise }\end{cases}
$$

The optimal strategies $\sigma_{\mathbf{b}_{j}}^{*}(t) \mid\left\{\mathbf{b}_{i}\right\}$ of the buyer $\mathbf{b}_{j}$ when it is believed to be $\mathbf{b}_{i}$ are:

- If $\iota(T)=\mathbf{b}$, accept $y$ if $y \leq \min \left\{\left(x_{\mathbf{b}_{i}}^{*}(t)\right)_{\leftarrow \mathbf{b}_{j}}, \mathrm{RP}_{j}\right\}$. Otherwise, offer $\min \left\{x_{\mathbf{b}_{i}}^{*}(t), \mathrm{RP}_{j}\right\}$.

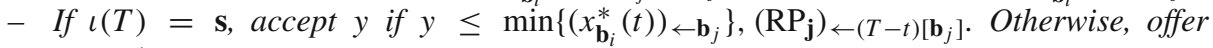

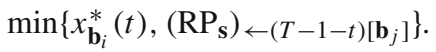

Proof Case 1 ( $\mathbf{b}_{i}$ is believed to be $\mathbf{b}_{j}$ ). If the seller offers $x_{\mathbf{b}_{j}}^{*}(t-1)$, buyer $\mathbf{b}_{i}$ 's optimal strategy is to accept it as the minimum price that the seller would accept at time $t+1$, i.e., $x_{\mathbf{b}_{j}}^{*}(t)$, gives $\mathbf{b}_{i}$ a utility lesser than $x_{\mathbf{b}_{j}}^{*}(t-1)$ since $\left(x_{\mathbf{b}_{j}}^{*}(t)\right)_{\leftarrow \mathbf{b}_{i}}>\left(x_{\mathbf{b}_{j}}^{*}(t)\right)_{\leftarrow \mathbf{b}_{j}}=x_{\mathbf{b}_{j}}^{*}(t-1)$. If the seller acts off the equilibrium path and offers a price $y$ lower than $x_{\mathbf{b}_{j}}^{*}(t-1)$, the optimal strategy of $\mathbf{b}_{i}$ is obviously to accept $y$. If the seller offers a price $y$ greater than $x_{\mathbf{b}_{j}}^{*}(t-1)$, the 
optimal strategy of $\mathbf{b}_{i}$ is to accept $y$ only if $y \leq\left(x_{\mathbf{b}_{j}}^{*}(t)\right)_{\leftarrow \mathbf{b}_{i}}$, otherwise $\mathbf{b}_{i}$ 's optimal strategy is to reject $y$ and to offer $x_{\mathbf{b}_{j}}^{*}(t)$. Note that $x_{\mathbf{b}_{i}}^{*}(t) \leq \mathrm{RP}_{i}$ and $x_{\mathbf{b}_{j}}^{*}(t) \leq \mathrm{RP}_{i}$.

Case 2 ( $\mathbf{b}_{j}$ is believed to be $\mathbf{b}_{i}$ ). This case is more complicated as seller's optimal offer $x_{\mathbf{b}_{i}}^{*}(t-1)$ on its equilibrium path is not acceptable to $\mathbf{b}_{j}$ as when $\mathbf{b}_{j}$ offers $x_{\mathbf{b}_{j}}^{*}(t)$ at time $t,\left(x_{\mathbf{b}_{i}}^{*}(t)\right)_{\leftarrow \mathbf{b}_{j}}<\left(x_{\mathbf{b}_{i}}^{*}(t)\right)_{\leftarrow \mathbf{b}_{i}}=x_{\mathbf{b}_{i}}^{*}(t-1)$. In addition, $\mathbf{b}_{j}$ may not offer $x_{\mathbf{b}_{i}}^{*}(t)$ if it is advantageous to wait for the agreement at time $T$. There are two situations: (1) $\iota(T)=\mathbf{b}$. In this case, $\mathbf{s}$ will propose $\mathrm{RP}_{i}$ at time $T-1$, which is not acceptable to buyer $\mathbf{b}_{j}$ as $\mathrm{RP}_{i}$ is higher than $\mathbf{b}_{j}$ 's reserve price. Therefore, $\mathbf{b}_{j}$ 's optimal offer at time $t \operatorname{is} \min \left\{x_{\mathbf{b}_{i}}^{*}(t), \operatorname{RP}_{j}\right\}$. Note that $x_{\mathbf{b}_{j}}^{*}(t)$ is always not acceptable to $\mathbf{s}$. (2) $\iota(T)=\mathbf{s}$. In this case, $\mathbf{b}_{j}$ will propose $\mathrm{RP}_{\mathbf{s}}$ at time $T-1$, which will be accepted by seller $\mathbf{s}$ at time $T$. Therefore, $\mathbf{b}_{j}$ 's optimal offer at time $t$ is $\min \left\{x_{\mathbf{b}_{i}}^{*}(t),\left(\mathrm{RP}_{\mathbf{s}}\right)_{\leftarrow(T-1-t)\left[\mathbf{b}_{j}\right]}\right\}$.

\section{The buyer's equilibrium offer}

This section focuses on computing the buyer's equilibrium offer at a continuation game $\Gamma\left(t, \Delta_{\mathbf{b}}\right)$ such that $\iota(t)=\mathbf{b}$. If $t=T$, it is the buyer agent's dominant strategy to accept any offer which is not worse than its reserve price. At time $t=T-1$, different buyer types' optimal offer is $\mathrm{RP}_{\mathbf{S}}$ since seller $\mathbf{s}$ will accept the offer at time $T$. If $\left|\Delta_{\mathbf{b}}\right|=1$, agents' equilibrium strategies are the equilibrium strategies of the corresponding complete information bargaining discussed in Sect. 2. When $\left|\Delta_{\mathbf{b}}\right|>1$ at time $t<T-1$, buyer types have multiple choice rules and we need to consider the equilibrium strategies for each choice rule. There could be multiple equilibrium strategies for a choice rule since there could be multiple sequential equilibria for the continuation game with a reasonable belief starting from time $t+1$. In the rest of this section, we show how to compute different buyer types' equilibrium strategies given agents' equilibrium strategies of the continuation game with different beliefs and construct agents' belief systems.

\subsection{Pooling choice rule}

Here we consider agents' equilibrium strategies when $\mathbf{b}$ employs a pooling choice rule at a continuation game $\Gamma\left(t, \Delta_{\mathbf{b}}\right)$. Since all buyer types will behave in the same way, seller $\mathbf{s}$ will not change its belief after observing the buyer's equilibrium offer. Thus, we need to consider all sequential equilibria $\mathbf{S E}\left(\Delta_{\mathbf{b}}, t+1\right)$ of the continuation game with belief $\Delta_{\mathbf{b}}$ at time $t+1$. If $\mathbf{S E}\left(\Delta_{\mathbf{b}}, t+1\right)=\emptyset$, there is no sequential equilibrium for this choice rule. Otherwise, for each sequential equilibrium $\wp \in \mathbf{S E}\left(\Delta_{\mathbf{b}}, t+1\right)$, we compute buyer types' optimal offer and check the satisfaction of equilibrium existence conditions.

First we consider the accepting pooling choice rule. Let $e_{\mathbf{s}}^{t+1} \mid \Delta_{\mathbf{b}}$ be $\mathbf{s}$ 's equivalent offer at time $t+1$ given the belief $\Delta_{\mathbf{b}}$ in the sequential equilibrium $\wp$. At time $t+1$, the equilibrium strategy of $\mathbf{s}$ is that $\mathbf{s}$ will accept any offer $y$ if $y \geq\left(e_{\mathbf{s}}^{t+1} \mid \Delta_{\mathbf{b}}\right)_{\leftarrow \mathbf{s}}$. Therefore, the equilibrium offer of buyer $\mathbf{b}_{i} \in \Delta_{\mathbf{b}}$ at time $t$ is $\left(e_{\mathbf{s}}^{t+1} \mid \Delta_{\mathbf{b}}\right) \leftarrow \mathbf{s} .{ }^{5}$ The corresponding offer update rule is the following:

$$
\mu(t+1)= \begin{cases}\Delta_{\mathbf{b}} & \text { if } \sigma_{\mathbf{b}}(t)=\text { offer }\left(e_{\mathbf{s}}^{t+1} \mid \Delta_{\mathbf{b}}\right) \leftarrow \mathbf{s} \\ \left\{\mathbf{b}_{h}\left(\Delta_{\mathbf{b}}\right)\right\} & \text { otherwise }\end{cases}
$$

\footnotetext{
5 We assume that buyer types are "cooperatively selfish" in the sense that when they are making the same acceptable offer, the will choose the lowest acceptable price.
} 
If buyer $\mathbf{b}_{i} \in \Delta_{\mathbf{b}}$ deviates from offering $\left(e_{\mathbf{S}}^{t+1} \mid \Delta_{\mathbf{b}}\right)_{\leftarrow \mathbf{s}}$, it will be believed to be of type $\mathbf{b}_{h}\left(\Delta_{\mathbf{b}}\right)$. Following Theorem 11, when a buyer $\mathbf{b}_{i}$ is believed to be of type $\mathbf{b}_{h}\left(\Delta_{\mathbf{b}}\right)$ which has a reserve price no less than $\mathrm{RP}_{i}, \mathbf{b}_{i}$ 's optimal offer at time $t$ is $x_{\mathbf{b}_{i}}^{*}(t) \mid\left\{\mathbf{b}_{h}\left(\Delta_{\mathbf{b}}\right)\right\}$. Thus, the condition of equilibrium existence needed to be checked is $e_{\mathbf{b}_{i}}^{t}\left|\Delta_{\mathbf{b}} \leq x_{\mathbf{b}_{i}}^{*}(t)\right|\left\{\mathbf{b}_{h}\left(\Delta_{\mathbf{b}}\right)\right\}$ for all $\mathbf{b}_{i} \in \Delta_{\mathbf{b}}$. If the equilibrium existence conditions are satisfied, there is a sequential equilibrium with buyer types' offer $\left(e_{\mathbf{S}}^{t+1} \mid \Delta_{\mathbf{b}}\right)_{\leftarrow \mathbf{s}}$ and $\wp$ as the sequential equilibrium for the continuation game from time $t+1$. The sequential equilibrium will be added to $\mathbf{S E}\left(\Delta_{\mathbf{b}}, t\right)$. Buyer $\mathbf{b}_{i}$ 's equilibrium bargaining outcome in this equilibrium is $E B O\left(\mathbf{b}_{i},\left(e_{\mathbf{s}}^{t+1} \mid \Delta_{\mathbf{b}}\right) \leftarrow \mathbf{s}, t\right)$ $=\left(\left(e_{\mathbf{s}}^{t+1} \mid \Delta_{\mathbf{b}}\right)_{\leftarrow \mathbf{s}}, t+1\right)$ since $\left(e_{\mathbf{s}}^{t+1} \mid \Delta_{\mathbf{b}}\right)_{\leftarrow \mathbf{s}}$ is acceptable to the seller. Thus buyer $\mathbf{b}_{i}$ 's equivalent offer is $e_{\mathbf{b}_{i}}^{t} \mid \Delta_{\mathbf{b}}=\left(e_{\mathbf{s}}^{t+1} \mid \Delta_{\mathbf{b}}\right) \leftarrow \mathbf{s}$.

Then we consider the rejecting pooling choice rule. By definition, all buyer types $\Delta_{\mathbf{b}}$ will make an offer (i.e., -1$)$ that will be rejected by the seller. Buyer $\mathbf{b}_{i}$ 's equilibrium bargaining outcome is the bargaining outcome in the sequential equilibrium $\wp$, i.e., $E B O\left(\mathbf{b}_{i},-1\right.$, $t)=E B O\left(\mathbf{b}_{i}, \wp\right)$. Thus buyer $\mathbf{b}_{i}$ 's equivalent offer is $e_{\mathbf{b}_{i}}^{t} \mid \Delta_{\mathbf{b}}=\rho\left(\mathbf{b}_{i}, t, E B O\left(\mathbf{b}_{i}, \wp\right)\right)$. We can also derive $e_{\mathbf{b}_{i}}^{t} \mid \Delta_{\mathbf{b}}$ in the following way. If according to the sequential equilibrium $\wp$, seller s's equilibrium offer $x^{t+1} \mid \Delta_{\mathbf{b}}$ at time $t+1$ is $\varpi$, buyer $\mathbf{b}_{i}$ 's equivalent offer at time $t$ is then $e_{\mathbf{b}_{i}}^{t} \mid \Delta_{\mathbf{b}}=\left(e_{\mathbf{b}_{i}}^{t+2} \mid \Delta_{\mathbf{b}}\right) \leftarrow 2\left[\mathbf{b}_{i}\right]$ where $e_{\mathbf{b}_{i}}^{t+2} \mid \Delta_{\mathbf{b}}$ is $\mathbf{b}_{i}$ 's equivalent offer at time $t+2$. If $x^{t+1} \mid \Delta_{\mathbf{b}} \neq \varpi$, buyer $\mathbf{b}_{i}$ 's equivalent offer $e_{\mathbf{b}_{i}}^{t} \mid \Delta_{\mathbf{b}}$ at time $t$ is

- $e_{\mathbf{b}_{i}}^{t} \mid \Delta_{\mathbf{b}}=\left(x^{t+1} \mid \Delta_{\mathbf{b}}\right) \leftarrow \mathbf{b}_{i}$ if according to the sequential equilibrium $\wp$, s's equilibrium offering price $x^{t+1} \mid \Delta_{\mathbf{b}}$ at time $t+1$ will be accepted by buyer type $\mathbf{b}_{i}$

- $e_{\mathbf{b}_{i}}^{t} \mid \Delta_{\mathbf{b}}=\left(e_{\mathbf{b}_{i}}^{t+2} \mid \Delta_{\mathbf{b}}^{\prime}\right) \leftarrow 2\left[\mathbf{b}_{i}\right]$ if according to the sequential equilibrium $\wp$, buyer type $\mathbf{b}_{i}$ will reject s's equilibrium offering price $x^{t+1} \mid \Delta_{\mathbf{b}}$ at time $t+1$ where $\Delta_{\mathbf{b}}^{\prime}$ is the set of buyer types that will reject s's offer at time $t+1$

The corresponding offer update rule is the following:

$$
\mu(t+1)= \begin{cases}\Delta_{\mathbf{b}} & \text { if } \sigma_{\mathbf{b}}(t)=\text { offer }-1 \\ \left\{\mathbf{b}_{h}\left(\Delta_{\mathbf{b}}\right)\right\} & \text { otherwise }\end{cases}
$$

If buyer $\mathbf{b}$ deviates from offering -1 at time $t$, it will be treated as buyer type $\mathbf{b}_{h}\left(\Delta_{\mathbf{b}}\right)$ and the equilibrium existence condition is $e_{\mathbf{b}_{i}}^{t}\left|\Delta_{\mathbf{b}} \leq x_{\mathbf{b}_{i}}^{*}(t)\right|\left\{\mathbf{b}_{h}\left(\Delta_{\mathbf{b}}\right)\right\}$ for all $\mathbf{b}_{i} \in \Delta_{\mathbf{b}}$.

\subsection{Separating choice rule}

Next we consider agents' equilibrium strategies at a continuation game $\Gamma\left(t, \Delta_{\mathbf{b}}\right)$ when buyer b employs the separating choice rule where buyer types $\Delta_{\mathbf{b}}^{a}$ make an acceptable offer while buyer types $\Delta_{\mathbf{b}}^{r}$ make a rejectable offer -1 . For this choice rule, the reasonable beliefs of its continuation game are $\Delta_{\mathbf{b}}^{a}$ and $\Delta_{\mathbf{b}}^{r}$. If one of the continuation games has no sequential equilibrium, there is no sequential equilibrium for this choice rule. We show how to compute agents' equilibrium strategies at time $t$ given a sequential equilibrium $\wp^{a} \in \mathbf{S E}\left(\Delta_{\mathbf{b}}^{a}, t+1\right)$ and a sequential equilibrium $\wp^{r} \in \mathbf{S E}\left(\Delta_{\mathbf{b}}^{r}, t+1\right)$.

Let $e_{\mathbf{S}}^{t+1} \mid \Delta_{\mathbf{b}}^{a}$ be s's equivalent offer at time $t+1$ in the equilibrium $\wp^{a}$. Let $e_{\mathbf{s}}^{t+1} \mid \Delta_{\mathbf{b}}^{r}$ $\left(x^{t+1} \mid \Delta_{\mathbf{b}}^{r}\right.$, respectively) be $\mathbf{s}$ 's equivalent offer (equilibrium offer, respectively) at time $t+1$ in the equilibrium $\wp^{r}$. Similar to the pooling acceptance choice rule, the optimal offer of buyer types $\Delta_{\mathbf{b}}^{a}$ at time $t$ is $\left(e_{\mathbf{s}}^{t+1} \mid \Delta_{\mathbf{b}}^{a}\right) \leftarrow \mathbf{s}$. Accordingly, buyer $\mathbf{b}_{i} \in \Delta_{\mathbf{b}}^{a}$, s equivalent offer is $e_{\mathbf{b}_{i}}^{t} \mid \Delta_{\mathbf{b}}=\left(e_{\mathbf{s}}^{t+1} \mid \Delta_{\mathbf{b}}^{a}\right)_{\leftarrow \mathbf{s}}$ since its equilibrium bargaining outcome is $\left(\left(e_{\mathbf{s}}^{t+1} \mid \Delta_{\mathbf{b}}^{a}\right)_{\leftarrow \mathbf{s}}, t+1\right)$. By convention, the equilibrium offer of buyer type $\mathbf{b}_{j} \in \Delta_{\mathbf{b}}^{r}$ at time $t$ is -1 . Buyer $\mathbf{b}_{j}$ 's equilibrium bargaining outcome is the bargaining outcome $E B O\left(\mathbf{b}_{j}, \wp^{r}\right)$ in the sequential 
equilibrium $\wp^{r}$. Thus buyer $\mathbf{b}_{j} \in \Delta_{\mathbf{b}}^{r}$ 's equivalent offer is $e_{\mathbf{b}_{j}}^{t} \mid \Delta_{\mathbf{b}}=\rho\left(\mathbf{b}_{j}, t, E B O\left(\mathbf{b}_{j}, \wp^{r}\right)\right)$. We can also compute $e_{\mathbf{b}_{j}}^{t} \mid \Delta_{\mathbf{b}}$ by considering the following two situations:

- If $x^{t+1} \mid \Delta_{\mathbf{b}}^{r} \neq \varpi$, the equivalent offer of buyer $\mathbf{b}_{j} \in \Delta_{\mathbf{b}}^{r}$ at time $t$ is (1) $e_{\mathbf{b}_{j}}^{t} \mid \Delta_{\mathbf{b}}=$ $\left(x^{t+1} \mid \Delta_{\mathbf{b}}^{r}\right) \leftarrow \mathbf{b}_{j}$ if according to the sequential equilibrium $\wp^{r}, \mathbf{s}$ 's equilibrium offering price $x^{t+1} \mid \Delta_{\mathbf{b}}^{r}$ at time $t+1$ will be accepted by buyer type $\mathbf{b}_{j} ; \operatorname{or}(2) e_{\mathbf{b}_{j}}^{t} \mid \Delta_{\mathbf{b}}=\left(e_{\mathbf{b}_{j}}^{t+2} \mid \Delta_{\mathbf{b}}^{r^{\prime}}\right) \leftarrow 2\left[\mathbf{b}_{j}\right]$ if according to the sequential equilibrium $\wp^{r}$, buyer type $\mathbf{b}_{j}$ will reject $\mathbf{s}$ 's equilibrium offering price $x^{t+1} \mid \Delta_{\mathbf{b}}^{r}$ at time $t+1$ where $\Delta_{\mathbf{b}}^{r^{\prime}} \subseteq \Delta_{\mathbf{b}}^{r}$ is the set of buyer types that will reject s's offer at time $t+1$.

- If $x^{t+1} \mid \Delta_{\mathbf{b}}^{r}=\varpi$, buyer $\mathbf{b}_{j} \in \Delta_{\mathbf{b}}^{r}$ 's equivalent offer at time $t$ is then $e_{\mathbf{b}_{j}}^{t} \mid \Delta_{\mathbf{b}}=$ $\left(e_{\mathbf{b}_{j}}^{t+2} \mid \Delta_{\mathbf{b}}^{r}\right) \leftarrow 2\left[\mathbf{b}_{j}\right]$ where $e_{\mathbf{b}_{j}}^{t+2} \mid \Delta_{\mathbf{b}}^{r}$ is $\mathbf{b}_{j}$ 's equivalent offer at time $t+2$.

Seller $\mathbf{s}$ will update its belief to $\Delta_{\mathbf{b}}^{a}$ when it receives an offer $\left(e_{\mathbf{s}}^{t+1} \mid \Delta_{\mathbf{b}}^{a}\right)_{\leftarrow \mathbf{s}}$. If it receives an offer -1 , it will update its belief to $\Delta_{\mathbf{b}}^{r}$. Otherwise, it will update its belief to $\mathbf{b}_{h}\left(\Delta_{\mathbf{b}}\right)$. The existence of such an equilibrium depends on the following conditions:

- Any buyer type $\mathbf{b}_{i} \in \Delta_{\mathbf{b}}^{a}$ has no incentive to behave as any buyer type $\mathbf{b}_{j} \in \Delta_{\mathbf{b}}^{r}$. If $\mathbf{b}_{i}$ pretends to be $\mathbf{b}_{j}$, it will offer -1 at time $t$ and its equilibrium bargaining outcome will be $E B O\left(\mathbf{b}_{j},-1, t\right)=E B O\left(\mathbf{b}_{j}, \wp^{r}\right)$. Therefore, this condition requires that $U_{\mathbf{b}_{i}}\left(E B O\left(\mathbf{b}_{i},\left(e_{\mathbf{s}}^{t+1} \mid \Delta_{\mathbf{b}}^{a}\right) \leftarrow \mathbf{s}, t\right)\right) \geq U_{\mathbf{b}_{i}}\left(E B O\left(\mathbf{b}_{j}, \wp^{r}\right)\right)$ or equivalently, $\left(e_{\mathbf{s}}^{t+1} \mid \Delta_{\mathbf{b}}^{a}\right) \leftarrow \mathbf{s}$ $\leq \rho\left(\mathbf{b}_{i}, t, E B O\left(\mathbf{b}_{j}, \wp^{r}\right)\right)$.

- Any buyer type $\mathbf{b}_{j} \in \Delta_{\mathbf{b}}^{r}$ must have no incentive to behave as $\mathbf{b}_{i} \in \Delta_{\mathbf{b}}^{a}$. If $\mathbf{b}_{j}$ behaves as $\mathbf{b}_{i}$, it will offer $\left(e_{\mathbf{s}}^{t+1} \mid \Delta_{\mathbf{b}}^{a}\right)_{\leftarrow \mathbf{s}}$ at time $t$ and the offer will be accepted. $\mathbf{b}_{j}$ will not choose to behave as $\mathbf{b}_{i}$ if $U_{\mathbf{b}_{j}}\left(E B O\left(\mathbf{b}_{j}, \wp^{r}\right) \geq U_{\mathbf{b}_{j}}\left(E B O\left(\mathbf{b}_{j},\left(e_{\mathbf{s}}^{t+1} \mid \Delta_{\mathbf{b}}^{a}\right)_{\leftarrow \mathbf{s}}, t\right)\right)\right)$ or equivalently, $\rho\left(\mathbf{b}_{j}, t, E B O\left(\mathbf{b}_{j}, \wp^{r}\right)\right) \leq\left(e_{\mathbf{s}}^{t+1} \mid \Delta_{\mathbf{b}}^{a}\right)_{\leftarrow \mathbf{s}}$.

- No buyer type has an incentive to offer a price different from the above two equilibrium offers. If a buyer type $\mathbf{b}_{i} \in \Delta_{\mathbf{b}}$ offers a price different from $\left(e_{\mathbf{s}}^{t+1} \mid \Delta_{\mathbf{b}}^{a}\right)_{\leftarrow \mathbf{s}}$ and -1 , it will be treated as buyer type $\mathbf{b}_{h}\left(\Delta_{\mathbf{b}}\right)$ and its optimal offer at time $t$ is then $x_{\mathbf{b}_{i}}^{*}(t) \mid\left\{\mathbf{b}_{h}\left(\Delta_{\mathbf{b}}\right)\right\}$. Buyer type $\mathbf{b}_{i}$ will not choose to act off the equilibrium path if $e_{\mathbf{b}_{i}}^{t}\left|\Delta_{\mathbf{b}} \leq x_{\mathbf{b}_{i}}^{*}(t)\right|\left\{\mathbf{b}_{h}\left(\Delta_{\mathbf{b}}\right)\right\}$.

If all the three conditions are satisfied, buyer types' optimal offers, the belief update rule, and the sequential equilibria $\wp^{a}$ and $\wp^{r}$ for the continuation game starting from time $t+1$ consists of a sequential equilibrium for the continuation game $\Gamma\left(t, \Delta_{\mathbf{b}}\right)$.

\section{The seller's equilibrium offer}

This section discusses how to compute the seller's equilibrium offer at a continuation game $\Gamma\left(t, \Delta_{\mathbf{b}}\right)$ such that $\iota(t)=\mathbf{s}$. If $t=T$, it is the seller's dominant strategy to accept any offer which is not worse than its reserve price. At time $t=T-1$, seller $\mathbf{s}$ has multiple choices, each for one buyer type in $\Delta_{\mathbf{b}}$. The optimal offer of seller $\mathbf{s}$ for buyer type $\mathbf{b}_{i} \in \Delta_{\mathbf{b}}$ is $\mathrm{RP}_{i}$, which gives seller $\mathbf{s}$ an expected utility $E U_{\mathbf{s}}\left(\mathrm{RP}_{i}, T-1\right)=\sum_{\mathbf{b}_{j} \in \Delta_{\mathbf{b}}, \mathrm{RP}_{j} \geq \mathrm{RP}_{i}} \omega_{\mathbf{b}_{j}}\left(\Delta_{\mathbf{b}}\right) U_{\mathbf{s}}\left(\mathrm{RP}_{i}, T\right)$ since $\mathrm{RP}_{i}$ is only acceptable to a buyer type with a reserve price no less than $\mathrm{RP}_{i}$. The optimal offer of $\mathbf{s}$ at time $T-1$ is $y=\arg \max _{y \in\left\{\mathrm{RP}_{i} \mid \mathbf{b}_{i} \in \Delta_{\mathbf{b}}\right\}} E U_{\mathbf{s}}(y, T-1)$ and its equivalent offer is $e_{\mathbf{s}}^{T-1} \mid \Delta_{\mathbf{b}}$ such that $U_{\mathbf{s}}\left(e_{\mathbf{s}}^{T-1} \mid \Delta_{\mathbf{b}}, T\right)=E U_{\mathbf{s}}(y, T-1)$. If $\left|\Delta_{\mathbf{b}}\right|=1$, agents' equilibrium strategies are the equilibrium strategies of the corresponding complete information bargaining discussed in Sect. 2.

Our idea of computing the seller's equilibrium offer given a belief $\Delta_{\mathbf{b}}\left(\left|\Delta_{\mathbf{b}}\right|>1\right)$ at time $t<T-1$ is the following. We consider all possible reject update rules and for each 
reject update rule, we compute all the sequential equilibria for the continuation game with beliefs corresponding to the reject update rule. Then for each sequential equilibrium for a reject update rule, we compute the seller's optimal offer and check the equilibrium existence conditions. Theorem 4 suggests that we only need to consider reject update rules in which buyer types with higher reserve prices accept the seller's equilibrium offer while buyer types with lower reserve prices reject the seller's equilibrium offer. Thus we only need to consider a restricted set of beliefs for the continuation game.

We consider a reject update rule in which buyer types $\Delta_{\mathbf{b}}^{\prime}$ will reject the seller's offer and buyer types $\Delta_{\mathbf{b}}-\Delta_{\mathbf{b}}^{\prime}$ will accept the seller's offer such that such that $\mathrm{RP}_{i}>\mathrm{RP}_{j}$ for any $\mathbf{b}_{i} \in \Delta_{\mathbf{b}}-\Delta_{\mathbf{b}}^{\prime}$ and $\mathbf{b}_{j} \in \Delta_{\mathbf{b}}^{\prime}$. We first compute all the sequential equilibria $\mathbf{S E}\left(\Delta_{\mathbf{b}}^{\prime}, t+1\right)$ for the continuation game with belief $\Delta_{\mathbf{b}}^{\prime}$ starting from time $t+1$. If there is no sequential equilibrium for the continuation game $\Gamma\left(t+1, \Delta_{\mathbf{b}}^{\prime}\right)$, there is no sequential equilibrium for this reject update rule. Otherwise, for each sequential equilibrium $\wp \in \mathbf{S E}\left(\Delta_{\mathbf{b}}^{\prime}, t+1\right)$, we check whether there exists a price $x$ such that the price, the reject update rule, and the sequential equilibrium $\wp$ constitute a sequential equilibrium for the continuation game $\Gamma\left(t, \Delta_{\mathbf{b}}\right)$.

A price $x$, a reject update rule, and a sequential equilibrium $\wp$ constitute a sequential equilibrium if and only if the following three conditions are satisfied (assume that $\mathbf{b}_{i} \in \Delta_{\mathbf{b}}-\Delta_{\mathbf{b}}^{\prime}$ and $\mathbf{b}_{j} \in \Delta_{\mathbf{b}}^{\prime}$ where buyer types $\Delta_{\mathbf{b}}^{\prime}$ will reject the seller's offer $x$ ):

1. $\mathbf{b}_{i}$ is willing to accept the offer $x$ and does not want to behave as $\mathbf{b}_{j}$. That is, for any $\mathbf{b}_{i} \in \Delta_{\mathbf{b}}-\Delta_{\mathbf{b}}^{\prime}$ and $\mathbf{b}_{j} \in \Delta_{\mathbf{b}}^{\prime}, U_{\mathbf{b}_{i}}(x, t+1) \geq U_{\mathbf{b}_{i}}\left(E B O\left(\mathbf{b}_{j}, \wp\right)\right)$ where $E B O\left(\mathbf{b}_{j}, \wp\right)$ is the $\mathbf{b}_{i}$ 's equilibrium bargaining outcome when it behaves as $\mathbf{b}_{j}$. This condition can be reformulated as $x \leq \min _{\mathbf{b}_{i} \in \Delta_{\mathbf{b}}-\Delta_{\mathbf{b}}^{\prime}, \mathbf{b}_{j} \in \Delta_{\mathbf{b}}^{\prime}} \rho\left(\mathbf{b}_{i}, t, E B O\left(\mathbf{b}_{j}, \wp\right)\right)$, which provides an upper bound for seller's offering price $x$. Intuitively, if the offering price $x$ is too high (e.g., higher than $\mathrm{RP}_{i}$ ), $\mathbf{b}_{i}$ can not accept the offering price.

2. $\mathbf{b}_{j}$ will reject the offer $x$. That is, each buyer type $\mathbf{b}_{j} \in \Delta_{\mathbf{b}}^{\prime}$ has no incentive to behave as $\mathbf{b}_{i}$, i.e., $U_{\mathbf{b}_{j}}(x, t+1)<U_{\mathbf{b}_{j}}\left(E B O\left(\mathbf{b}_{j}, \wp\right)\right)$. This condition can be rewritten as $x>\max _{\mathbf{b}_{j} \in \Delta_{\mathbf{b}}^{\prime}} \rho\left(\mathbf{b}_{j}, t, E B O\left(\mathbf{b}_{j}, \wp\right)\right)$, which provides a lower bound for the offering price $x$. Intuitively, if the offering price $x$ is very low (e.g., close to 0 ), $\mathbf{b}_{j}$ will choose to accept the favorite offer.

3. Seller $\mathbf{s}$ has no incentive to choose a price other than $x$ given the reject update rule and the sequential equilibrium $\wp$ of the continuation game $\Gamma\left(t+1, \Delta_{\mathbf{b}}^{\prime}\right)$;

The third condition requires that the price $x$ is seller's optimal offer given the reject update rule and the sequential equilibrium $\wp$ for the continuation game. Any buyer type can either accept the seller's offer $x$ or reject it and receive a bargaining outcome in the sequential equilibrium $\wp$ for the continuation game. Formally, buyer type $\mathbf{b}_{j} \in \Delta_{\mathbf{b}}^{\prime}$ will accept a price $x$ if and only if $x \leq \rho\left(\mathbf{b}_{j}, t, E B O\left(\mathbf{b}_{j}, \wp\right)\right)$. Buyer type $\mathbf{b}_{i} \in \Delta_{\mathbf{b}}-\Delta_{\mathbf{b}}^{\prime}$ will accept a price $x$ if and only if $x \leq \min _{\mathbf{b}_{j} \in \Delta_{\mathbf{b}}^{\prime}} \rho\left(\mathbf{b}_{i}, t, E B O\left(\mathbf{b}_{j}, \wp\right)\right)$. We can define the acceptance price $\phi\left(\mathbf{b}_{i}, \Delta_{\mathbf{b}}^{\prime}, \wp\right)$ of each buyer type $\mathbf{b}_{i} \in \Delta_{\mathbf{b}}$ given the sequential equilibrium $\wp$ as follows:

$$
\phi\left(\mathbf{b}_{i}, \Delta_{\mathbf{b}}^{\prime}, \wp\right)= \begin{cases}\rho\left(\mathbf{b}_{i}, t, E B O\left(\mathbf{b}_{i}, \wp\right)\right) & \text { if } \mathbf{b}_{i} \in \Delta_{\mathbf{b}}^{\prime} \\ \min _{\mathbf{b}_{j} \in \Delta_{\mathbf{b}}^{\prime}} \rho\left(\mathbf{b}_{i}, t, E B O\left(\mathbf{b}_{j}, \wp\right)\right) & \text { otherwise }\end{cases}
$$

Seller s's expected utility of making an offer $x$ given the sequential equilibrium $\wp$ is defined as

$$
E U_{\mathbf{s}}(x, t)=\sum_{\mathbf{b}_{i} \in \Delta_{\mathbf{b}}} \omega_{\mathbf{b}_{i}}\left(\Delta_{\mathbf{b}}\right) E U_{\mathbf{s}}\left(x, t, \mathbf{b}_{i}\right)
$$


where $E U_{\mathbf{s}}\left(x, t, \mathbf{b}_{i}\right)$ is seller s's utility if the buyer is of type $\mathbf{b}_{i}$, which is defined as

$$
E U_{\mathbf{s}}\left(x, t, \mathbf{b}_{i}\right)= \begin{cases}U_{\mathbf{S}}(x, t+1) & \text { if } x \leq \phi\left(\mathbf{b}_{i}, \Delta_{\mathbf{b}}^{\prime}, \wp\right) \\ U_{\mathbf{s}}\left(E B O\left(\mathbf{b}_{i}, \wp\right)\right) & \text { if } x>\phi\left(\mathbf{b}_{i}, \Delta_{\mathbf{b}}^{\prime}, \wp\right) \text { and } \mathbf{b}_{i} \in \Delta_{\mathbf{b}}^{\prime} \\ U_{\mathbf{s}}\left(\min _{\mathbf{b}_{j} \in \Delta_{\mathbf{b}}^{\prime}} \rho\left(\mathbf{b}_{i}, t, E B O\left(\mathbf{b}_{j}, \wp\right)\right), t+1\right) & \text { otherwise }\end{cases}
$$

It is easy to see that the optimal offer the seller should be either one buyer type's acceptance price or a price that will be rejected by all buyer types (i.e., $\varpi)$. If the seller's optimal offer $x$ satisfies the first two equilibrium existence conditions, there is a sequential equilibrium in which the seller offers price $x$ and buyer types $\Delta_{\mathbf{b}}^{\prime}$ will reject the offer with the sequential equilibrium $\wp$. If such a $x$ value does not exist, there is no sequential equilibrium given this reject update rule and the continuation game equilibrium $\wp$.

In addition to the above reject update rules under which at least one buyer type will choose to reject the offer, the seller can also make an offer such that it is all buyer types' equilibrium strategies to accept the offer. It is easy to see that the highest offer that will be accepted by all buyer types in equilibrium is $x=\min _{\mathbf{b}_{i} \in \Delta_{\mathbf{b}}}\left(x_{\mathbf{b}_{i}}^{*}(t+1) \mid \mathbf{b}_{h}\left(\Delta_{\mathbf{b}}\right)\right) \leftarrow \mathbf{b}_{i}$ since if a seller offers a price larger than $x$, at least one buyer type has an incentive to deviate from accepting the offer. If the buyer rejects $x$, the seller will update its belief to $\mathbf{b}_{h}\left(\Delta_{\mathbf{b}}\right)$. The acceptance price of buyer type $\mathbf{b}_{i}$ for this reject update rule is thus $\left(x_{\mathbf{b}_{i}}^{*}(t+1) \mid \mathbf{b}_{h}\left(\Delta_{\mathbf{b}}\right)\right)_{\leftarrow \mathbf{b}_{i}}$. If the optimal offer of the seller in this case is not acceptable to all the buyer types (i.e., the optimal offer is not $\left.\min _{\mathbf{b}_{i} \in \Delta_{\mathbf{b}}}\left(x_{\mathbf{b}_{i}}^{*}(t+1) \mid \mathbf{b}_{h}\left(\Delta_{\mathbf{b}}\right)\right) \leftarrow \mathbf{b}_{i}\right)$, there is no sequential equilibrium for this null reject update rule. Otherwise, there is a sequential equilibrium in which the seller will make an offer which will be accepted by all buyer types.

\section{Analysis of the approach}

This section analyzes the proposed approach. We first show that the proposed approach is sound and is complete. Then we experimentally show that there is at least one pure strategy sequential equilibrium in most games with moderate complexity. We also evaluate the running time of our algorithm. Finally, we give an example showing how our approach works.

\section{Theorem 12 Our algorithm can generate all pure strategy sequential equilibria.}

Proof Our algorithm is complete since at any decision making point, we consider (1) all sequential equilibria of the continuation game with different beliefs, (2) all choice rules when it is the buyer's turn to make an offer, and (3) all possible reject update rules if it is the seller's turn to make an offer. Furthermore, we use optimistic conjectures off the equilibrium path and, if an assessment is not an equilibrium with optimistic conjectures, then it cannot be of equilibrium with other conjectures.

If all equilibrium existence conditions are satisfied, agents' strategies and belief systems generated by our algorithm constitutes a sequential equilibrium. The sequential rationality is easily seen from the backward construction: agents' strategies at time $t$ are optimal in the continuation game starting from time $t$. Consistency can be proved by the assessment sequence $a_{n}=\left(\mu_{n}, \sigma_{n}\right)$ where $\sigma_{n}$ is the fully mixed strategy profile such that for the seller and buyer type $\mathbf{b}_{h}\left(\Delta_{\mathbf{b}}^{0}\right)$ there is probability $1-1 / n$ of performing the action prescribed by the equilibrium strategy profile and the remaining probability $1 / n$ is uniformly distributed among the other allowed actions, while for any other buyer type $\mathbf{b}_{i} \in \Delta_{\mathbf{b}}^{0}-\mathbf{b}_{h}\left(\Delta_{\mathbf{b}}^{0}\right)$, there is probability $1-1 / n^{T}$ of performing the action prescribed by the equilibrium strategy profile and the remaining probability $1 / n^{T}$ is uniformly distributed among the other allowed actions, 
Table 2 Simulation parameters

\begin{tabular}{ll}
\hline Parameters & Value range \\
\hline Deadline $(T)$ & $\{2, \ldots, 14\}$ \\
Number of buyer types $\left(\left|\Delta_{\mathrm{b}}^{0}\right|\right)$ & $\{2, \ldots, 9\}$ \\
Reserve price of buyer $\left(\mathrm{RP}_{i}\right)$ & {$[40,100]$} \\
Reserve price of seller $\left(\mathrm{RP}_{\mathrm{s}}\right)$ & {$[5,20]$} \\
Discounting factors $\left(\delta_{\mathrm{s}}, \delta_{\mathrm{b}}\right)$ & {$[0.5,1)$} \\
\hline
\end{tabular}

and $\mu_{n}$ is the system of beliefs obtained applying Bayes rule starting from the same prior probability distribution $P_{\mathbf{b}}^{0}$. As $n \rightarrow \infty$, the above mixed strategy profile converges to the equilibrium strategy profile. In addition, the beliefs generated by the mixed strategy profile converges to the prior probability distribution. Thus, the assessment is consistent.

Since we focus on pure strategy equilibrium, there may be no sequential equilibrium for some bargaining games. The non-existence problem of the equilibrium in pure strategies is critical since it may affect the applicability of alternating-offers protocol in realistic settings. Here we show one example which has no sequential equilibrium. The bargaining game with the following parameters: $T=2, \iota(0)=\mathbf{b}, \mathrm{RP}_{\mathrm{s}}=10, \mathrm{RP}_{1}=100, \mathrm{RP}_{2}=$ $40, \omega_{\mathbf{b}_{1}}^{0}=0.6, \omega_{\mathbf{b}_{2}}^{0}=0.4, \delta_{\mathbf{s}}=0.9$, and $\delta_{\mathbf{b}}=0.8$. First consider that the buyer is applying the pooling accepting choice rule at time 0 . With the initial belief, the seller will get a utility of $0.6(100-10) 0.9^{2}=43.74$ if it offers $\mathrm{RP}_{1}$ at time $t=1$ and will get a utility of $(40-10) 0.9^{2}=24.3$ if it offers $\mathrm{RP}_{2}$ at time $t=1$. Therefore, the optimal offer of the seller with the initial belief at $t=1$ is offering 100. Accordingly, the optimal offer of all buyer types is $(100)_{\leftarrow \mathbf{s}}=58.6$. Obviously, buyer $\mathbf{b}_{2}$ will deviate from it since the offering price 58.6 is higher than its reserve price 40 . Consider the pooling rejecting choice rule in which all buyer types offer -1 and buyer $\mathbf{b}_{1}$ 's equivalent offer is $(100)_{\leftarrow \mathbf{b}_{1}}=100$. In this case, buyer $\mathbf{b}_{1}$ has an incentive to offer $x_{\mathbf{b}_{1}}^{*}(0)=91$ which will be accepted by the seller since it will update its belief to $\left\{\mathbf{b}_{1}\right\}$ after receiving an offer other than 100 . The final choice rule for the buyer is the separating choice rule in which buyer $\mathbf{b}_{1}$ offers $x_{\mathbf{b}_{1}}^{*}(0)=91$ and buyer $\mathbf{b}_{2}$ offers -1 . However, buyer $\mathbf{b}_{1}$ has an incentive to offer -1 since at time $t=1$ the seller will offer $\mathrm{RP}_{2}=40$, which is better since $(40) \leftarrow \mathbf{b}_{1}=52<x_{\mathbf{b}_{1}}^{*}(0)=91$. Therefore, there is no sequential equilibrium for this bargaining game.

On the other hand, there may be multiple pure strategy sequential equilibria for each bargaining game and our algorithm can find all of them. Based on the computation reduction techniques, we just need to find sequential equilibria for at most $\left|\Delta_{\mathbf{b}}^{0}\right|$ continuation games $\Gamma\left(t, \Delta_{\mathbf{b}}\right)$ at time $t$ where $\Delta_{\mathbf{b}} \subseteq \Delta_{\mathbf{b}}^{0}$. Let $\Psi\left(t, \Delta_{\mathbf{b}}\right)$ be the maximum number of sequential equilibria for the continuation game $\Gamma\left(t, \Delta_{\mathbf{b}}\right)$. If $t=T-1$, it follows that $\Psi\left(t, \Delta_{\mathbf{b}}\right)=1$. Otherwise, we have the following: (1) If $\iota(t)=\mathbf{b}, \Psi\left(t, \Delta_{\mathbf{b}}\right)=\mathcal{O}\left(\left|\Delta_{\mathbf{b}}\right| \Psi\left(t+1, \Delta_{\mathbf{b}}\right)^{2}\right)$ since buyer types can try different choice rules and for each choice rule, we need to consider all the equilibrium combinations of at most two continuation games. (2) If $\iota(t)=\mathbf{s}, \Psi\left(t, \Delta_{\mathbf{b}}\right)<$ $\mathcal{O}\left(\left|\Delta_{\mathbf{b}}\right|^{2}\left(\Psi\left(t+1, \Delta_{\mathbf{b}}\right)\right)\right)$ since we need to consider $\left|\Delta_{\mathbf{b}}\right|+1$ reject update rules and for each reject update rule, we need to consider all the sequential equilibria for the corresponding continuation game. We can see that the number of sequential equilibria may double exponentially increase with the number $\left|\Delta_{\mathbf{b}}^{0}\right|$ of buyer types and the deadline $T$.

To evaluate the percentage of games with at least one pure strategy sequential equilibrium, we performed a series of experiments (using JAVA) in a variety of test environments and the parameters are given in Table 2 . In the experiments, the negotiation deadline is randomly 
selected from $\{2, \ldots, 14\}$, the number of buyer types is randomly selected from $\{2, \ldots, 9\}$ and the initial probability of each buyer type is set randomly. The reserve price of each buyer type is randomly selected from $[40,100]$ and the reserve price of the seller is randomly selected from $[5,20]$. Therefore, the reserve price of each buyer is always higher than the reserve price of the seller and the two agents have a negotiation space. Agents' discounting factors model how agents' utilities decrease with time. When discounting factors are smaller than 0.5 , agents' utilities drastically decrease with time. In order to make our setting more realistic, agents' discounting factors are randomly selected from $[0.5,1)$. The agent making the first offer is randomly determined. The above setting represents a wide range of scenarios.

Experimental results show that there is at least one sequential equilibrium in $\sim 99.7 \%$ of the bargaining games. Table 3 shows the average number of sequential equilibria (including both games with sequential equilibria and without sequential equilibria) and percentage of games with sequential equilibria in negotiation games with different deadlines and number of buyer types. For each combination of deadlines and number of buyer types, we randomly generated over 1,000 scenarios and computed the average values. We can see that the average number of sequential equilibria or the percentage of games with sequential equilibria increases with the increase of deadlines. Similarly, the average number of sequential equilibria slightly increase with the number of buyer types. It can be observed from Table 3 that when the deadline is longer than 2 and the number of buyer types is more than 3 , all scenarios have at least one sequential equilibrium. With more buyer types, there are more choice rules and reject update rules and potentially, there will be more sequential equilibria. However, more buyer types introduce more stringent equilibrium existence conditions since an equilibrium requires that each buyer type has an incentive to choose a different strategy.

Our algorithm can be treated as a tree based search algorithm, each node corresponding to a continuation game with certain belief. Consider the problem of solving a continuation game $\Gamma\left(t, \Delta_{\mathbf{b}}\right)$ at time $t$. If $\iota(t)=\mathbf{b}$, we need to consider $|\mathbf{b}|+1$ choice rules and thus we need to consider at most $2|\mathbf{b}|$ continuation games at time $t+1$. If $\iota(t)=\mathbf{s}$, we need to consider $\left|\Delta_{\mathbf{b}}\right|+1$ reject update rules and thus we need to consider at most $\left|\Delta_{\mathbf{b}}\right|+1$ continuation games at time $t+1$. The height of a tree is $T+1$. Therefore, the number of continuation games we need to solve is bounded by $\mathcal{O}\left(T\left|\Delta_{\mathbf{b}}^{0}\right|^{T+1}\right)$. In our backward induction approach, the equilibrium calculation for a continuation game with certain belief may be conducted multiple times. To avoid the repetition of equilibrium calculation in our approach, we stored known equilibrium strategies for each continuation game with certain belief. If there is no calculation repetition, the computational complexity of our approach is not higher than any other complete algorithm.

We experimentally evaluated the running time to our algorithm using the setting specified in Table 2. All experiments run on a PC with a $2.16 \mathrm{Ghz}$ Intel Pentium Dual processor and $2 \mathrm{~GB}$ of memory. Experimental results show that the algorithm's running time increases with the deadlines and the number of buyer types. Table 4 shows the average time of computing all the sequential equilibria in a bargaining game. The average running time is only about $12 \mathrm{~s}$ when the minimum deadline of the two agents is no higher than 14 and the number of buyer types is no more than 9 . We can find that the computation time increases drastically with the increase of both the negotiation deadline and the number of buyer types, which coincides with our analysis about the number of continuation games our algorithm has to solve.

In this paper we compute all the sequential equilibria for a bilateral bargaining game. The algorithm is much faster if we terminate our algorithm once it finds one sequential equilibrium. Table 5 shows the average computation time when we just compute one sequential equilibrium. The overall computation time for all the cases in Table 4 is drastically reduced as indicated by Table 5, with the more complicated cases showing a higher percentage of 


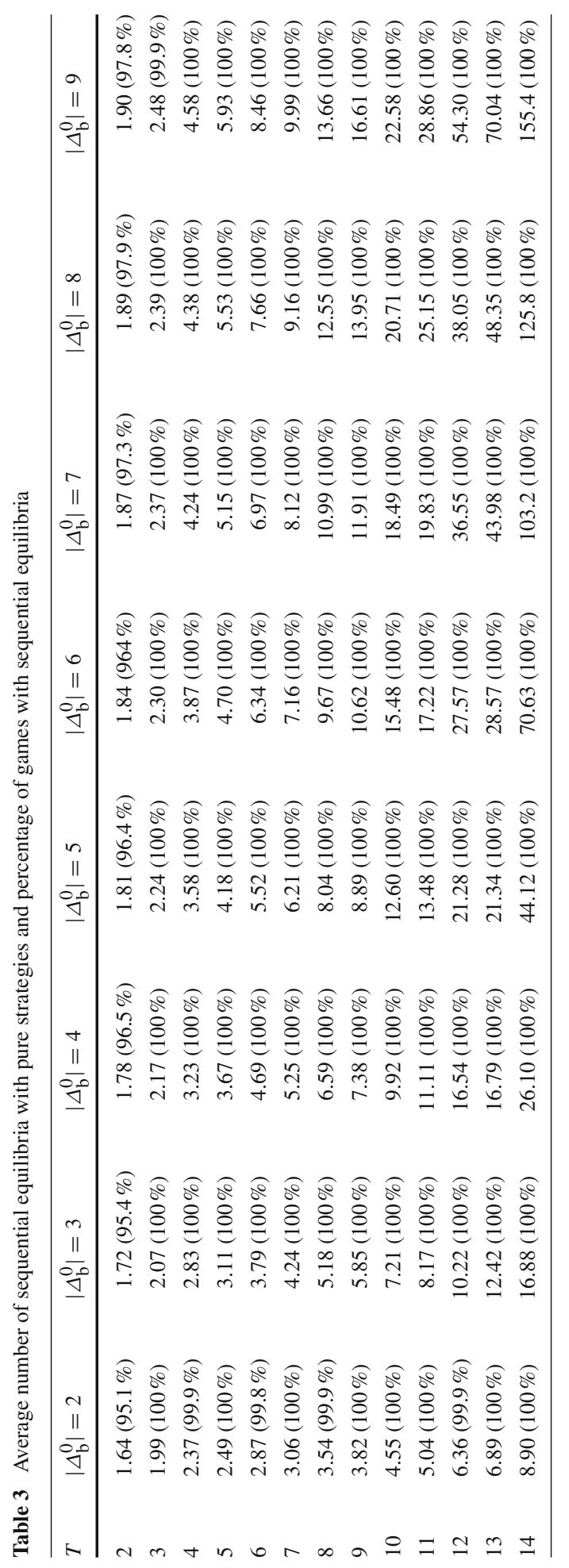


Table 4 Average computation time for computing all sequential equilibria (in seconds)

\begin{tabular}{lllllllll}
\hline$T$ & $\left|\Delta_{\mathrm{b}}^{0}\right|=2$ & $\left|\Delta_{\mathrm{b}}^{0}\right|=3$ & $\left|\Delta_{\mathrm{b}}^{0}\right|=4$ & $\left|\Delta_{\mathrm{b}}^{0}\right|=5$ & $\left|\Delta_{\mathrm{b}}^{0}\right|=6$ & $\left|\Delta_{\mathrm{b}}^{0}\right|=7$ & $\left|\Delta_{\mathrm{b}}^{0}\right|=8$ & $\left|\Delta_{\mathrm{b}}^{0}\right|=9$ \\
\hline 2 & 0.01 & 0.01 & 0.02 & 0.04 & 0.05 & 0.08 & 0.11 & 0.14 \\
3 & 0.02 & 0.05 & 0.11 & 0.21 & 0.37 & 0.60 & 0.95 & 1.38 \\
4 & 0.03 & 0.10 & 0.26 & 0.53 & 1.02 & 1.77 & 2.93 & 4.64 \\
5 & 0.05 & 0.17 & 0.45 & 0.97 & 1.90 & 3.39 & 5.78 & 9.35 \\
6 & 0.07 & 0.24 & 0.66 & 1.47 & 2.99 & 5.42 & 9.43 & 15.50 \\
7 & 0.08 & 0.33 & 0.90 & 2.07 & 4.22 & 7.85 & 13.82 & 23.09 \\
8 & 0.10 & 0.41 & 1.18 & 2.71 & 5.66 & 10.73 & 19.00 & 31.89 \\
9 & 0.12 & 0.51 & 1.46 & 3.45 & 7.27 & 13.78 & 24.90 & 42.61 \\
10 & 0.15 & 0.60 & 1.80 & 4.32 & 9.14 & 17.82 & 32.56 & 54.40 \\
11 & 0.17 & 0.73 & 2.16 & 5.24 & 11.39 & 22.33 & 41.32 & 70.60 \\
12 & 0.20 & 0.86 & 2.63 & 6.46 & 14.39 & 28.12 & 51.27 & 92.55 \\
13 & 0.23 & 1.02 & 3.09 & 7.75 & 17.28 & 36.79 & 69.48 & 119.52 \\
14 & 0.26 & 1.19 & 3.73 & 10.14 & 23.70 & 50.38 & 95.47 & 176.75 \\
\hline
\end{tabular}

Table 5 Average computation time when computing one equilibrium (in seconds)

\begin{tabular}{lllllllll}
\hline$T$ & $\left|\Delta_{\mathrm{b}}^{0}\right|=2$ & $\left|\Delta_{\mathrm{b}}^{0}\right|=3$ & $\left|\Delta_{\mathrm{b}}^{0}\right|=4$ & $\left|\Delta_{\mathrm{b}}^{0}\right|=5$ & $\left|\Delta_{\mathrm{b}}^{0}\right|=6$ & $\left|\Delta_{\mathrm{b}}^{0}\right|=7$ & $\left|\Delta_{\mathrm{b}}^{0}\right|=8$ & $\left|\Delta_{\mathrm{b}}^{0}\right|=9$ \\
\hline 2 & 0.01 & 0.01 & 0.02 & 0.03 & 0.04 & 0.06 & 0.09 & 0.11 \\
3 & 0.02 & 0.04 & 0.09 & 0.16 & 0.27 & 0.44 & 0.70 & 1.00 \\
4 & 0.03 & 0.07 & 0.17 & 0.34 & 0.63 & 1.06 & 1.73 & 2.72 \\
5 & 0.04 & 0.11 & 0.28 & 0.59 & 1.10 & 1.91 & 3.13 & 4.90 \\
6 & 0.05 & 0.15 & 0.38 & 0.79 & 1.52 & 2.70 & 4.61 & 7.12 \\
7 & 0.05 & 0.20 & 0.50 & 1.09 & 2.12 & 3.68 & 6.22 & 10.10 \\
8 & 0.06 & 0.23 & 0.59 & 1.29 & 2.49 & 4.53 & 7.70 & 12.74 \\
9 & 0.07 & 0.27 & 0.71 & 1.56 & 3.17 & 5.76 & 9.65 & 15.56 \\
10 & 0.08 & 0.29 & 0.78 & 1.78 & 3.49 & 6.39 & 10.81 & 17.36 \\
11 & 0.09 & 0.35 & 0.90 & 2.10 & 4.08 & 7.59 & 13.20 & 20.94 \\
12 & 0.10 & 0.37 & 0.95 & 2.19 & 4.51 & 7.91 & 14.57 & 22.73 \\
13 & 0.11 & 0.42 & 1.11 & 2.57 & 5.10 & 9.05 & 15.63 & 26.84 \\
14 & 0.12 & 0.44 & 1.16 & 2.60 & 5.25 & 9.44 & 16.87 & 29.82 \\
\hline
\end{tabular}

reduction. Most importantly, the effect of the deadline and the number of buyer types on the computation time is less significant, which is mainly due to the number of equilibria increases with the increase of the deadline and the number of buyer types (Table 3).

The algorithm for producing equilibrium strategies is a backward induction process, which starts from the continuation game with the initial belief at time $t=0$. Here we show an example of equilibrium calculation for the bargaining game with the following parameters: $T=3$, $\iota(0)=\mathbf{s}, \mathrm{RP}_{\mathbf{s}}=10, \mathrm{RP}_{1}=100, \mathrm{RP}_{2}=60, \mathrm{RP}_{3}=50, \omega_{\mathbf{b}_{1}}^{0}=0.25, \omega_{\mathbf{b}_{2}}^{0}=0.5, \omega_{\mathbf{b}_{3}}^{0}=$ $0.25, \delta_{\mathbf{s}}=0.8$, and $\delta_{\mathbf{b}}=0.6$. Before we start the backward induction process, we compute agents' equilibrium offers in complete information setting using the approach in Sect. 2 since we may use these equilibrium offers to construct agents' equilibrium strategies for the bargaining game. If buyer is of type $\mathbf{b}_{1}$, we have $x_{\mathbf{b}_{1}}^{*}(2)=100, x_{\mathbf{b}_{1}}^{*}(1)=82$, and $x_{\mathbf{b}_{1}}^{*}(0)=89.2$. 
If buyer is of type $\mathbf{b}_{2}$, we have $x_{\mathbf{b}_{2}}^{*}(2)=60, x_{\mathbf{b}_{2}}^{*}(1)=50.0$, and $x_{\mathbf{b}_{2}}^{*}(0)=54.0$. If buyer is of type $\mathbf{b}_{3}$, we have $x_{\mathbf{b}_{3}}^{*}(2)=50, x_{\mathbf{b}_{3}}^{*}(1)=42.0$, and $x_{\mathbf{b}_{3}}^{*}(0)=45.2$.

Now we use our algorithm to compute all the sequential equilibria. Since $\iota(0)=\mathbf{s}$, seller $\mathbf{s}$ will consider different reject update rules at time $t=0$. Therefore, we need to first compute all the sequential equilibria for the continuation game with reasonable beliefs $\left\{\mathbf{b}_{1}, \mathbf{b}_{2}, \mathbf{b}_{3}\right\}$, $\left\{\mathbf{b}_{2}, \mathbf{b}_{3}\right\}$, and $\left\{\mathbf{b}_{3}\right\}$ at time $t=1$. We show how to compute sequential equilibria for the continuation game with belief $\left\{\mathbf{b}_{1}, \mathbf{b}_{2}, \mathbf{b}_{3}\right\}$ at time $t=1$. At time $t=1$, $\mathbf{b}$ can apply different choice rules and we need to first compute all the sequential equilibria for the continuation game with beliefs $\left\{\mathbf{b}_{1}, \mathbf{b}_{2}, \mathbf{b}_{3}\right\},\left\{\mathbf{b}_{1}\right\},\left\{\mathbf{b}_{2}, \mathbf{b}_{3}\right\},\left\{\mathbf{b}_{1}, \mathbf{b}_{2}\right\}$, and $\left\{\mathbf{b}_{3}\right\}$ at time $t=2$. For the continuation game with belief $\left\{\mathbf{b}_{1}, \mathbf{b}_{2}, \mathbf{b}_{3}\right\}$ at time $t=2$, s can offer $\mathrm{RP}_{3}=50, \mathrm{RP}_{2}=60$, or $\mathrm{RP}_{1}=100$ : If it offers 50 , its expected utility is $(50-10) 0.8^{3}=20.48$; If it offers 60 , its expected utility is $0.75(60-10) 0.8^{3}=19.20$; If it offers 100 , its expected utility is $0.25(100-10) 0.8^{3}=11.52$. Thus, the optimal offer of $\mathbf{s}$ at time $t=3$ is 50 and the equivalent offer is $e_{\mathbf{s}}^{2} \mid\left\{\mathbf{b}_{1}, \mathbf{b}_{2}, \mathbf{b}_{3}\right\}=50.0$. When the belief is $\left\{\mathbf{b}_{2}, \mathbf{b}_{3}\right\}$, the optimal offer of $\mathbf{s}$ at time $t=3$ is 50 and the equivalent offer is $e_{\mathbf{s}}^{2} \mid\left\{\mathbf{b}_{2}, \mathbf{b}_{3}\right\}=50.0$. When the belief is $\left\{\mathbf{b}_{1}, \mathbf{b}_{2}\right\}$, the optimal offer of $\mathbf{s}$ at time $t=3$ is 60 and the equivalent offer is $e_{\mathbf{s}}^{2} \mid\left\{\mathbf{b}_{1}, \mathbf{b}_{2}\right\}=60.0$. For the pooling accepting choice rule at time $t=1$, the optimal offer of all buyer types is $\left(e_{\mathbf{s}}^{2} \mid\left\{\mathbf{b}_{1}, \mathbf{b}_{2}, \mathbf{b}_{3}\right\}\right)_{\leftarrow \mathbf{s}}=(50)_{\leftarrow \mathbf{s}}=42$ and no buyer has an incentive to deviate from it: if any buyer type chooses a different offer, it will be treated as buyer type $\mathbf{b}_{1}$ and its utility in the later negotiation is 0 . For the pooling rejecting choice rule at time $t=1$, all buyer types will offer -1 and no buyer has an incentive to deviate from it. For example, buyer $\mathbf{b}_{2}$ 's equivalent offer of offering -1 is $e_{\mathbf{b}_{2}}^{1} \mid\left\{\mathbf{b}_{1}, \mathbf{b}_{2}, \mathbf{b}_{3}\right\}=\left(e_{\mathbf{s}}^{2} \mid\left\{\mathbf{b}_{1}, \mathbf{b}_{2}, \mathbf{b}_{3}\right\}\right)_{\leftarrow \mathbf{b}_{2}}=(50) \leftarrow \mathbf{b}_{2}=54$. If buyer $\mathbf{b}_{2}$ deviates from offering -1 , it will be believed to be $\mathbf{b}_{1}$ and its optimal offer at time $t=1$ is then $x_{\mathbf{b}_{2}}^{*}(1) \mid\left\{\mathbf{b}_{1}\right\}=60$, which is higher than $e_{\mathbf{b}_{2}}^{1} \mid\left\{\mathbf{b}_{1}, \mathbf{b}_{2}, \mathbf{b}_{3}\right\}$. For the separating choice rule in which $\mathbf{b}_{1}$ makes an acceptable offer while the other two buyer types offer -1 , there is only one sequential equilibrium for the continuation game with beliefs $\left\{\mathbf{b}_{1}\right\}$ and $\left\{\mathbf{b}_{2}, \mathbf{b}_{3}\right\}$. Thus, the optimal offer of $\mathbf{b}_{1}$ is $x_{\mathbf{b}_{1}}^{1} \mid\left\{\mathbf{b}_{1}\right\}=x_{\mathbf{b}_{1}}^{*}(1)=82$. However, $\mathbf{b}_{1}$ has an incentive to behave as $\mathbf{b}_{2}$ or $\mathbf{b}_{3}$ since in the sequential equilibrium for the continuation game with beliefs $\left\{\mathbf{b}_{2}, \mathbf{b}_{3}\right\}$, s will make an offer 50 at time $t=2$ which can bring $\mathbf{b}_{1}$ a higher utility because $(50)_{\leftarrow \mathbf{b}_{1}}=70<82$. Therefore, there is no sequential equilibrium for this choice rule. However we can show that there is a sequential equilibrium for the separating choice rule in which $\mathbf{b}_{1}$ and $\mathbf{b}_{2}$ make an acceptable offer and their optimal offer is $\left(e_{\mathbf{s}}^{2} \mid\left\{\mathbf{b}_{1}, \mathbf{b}_{2}\right\}\right)_{\leftarrow \mathbf{s}}=(60)_{\leftarrow \mathbf{s}}=50$. There are totally 3 sequential equilibria for the continuation game with belief $\left\{\mathbf{b}_{1}, \mathbf{b}_{2}, \mathbf{b}_{3}\right\}$ at time $t=1$.

In the same way, we can compute the two sequential equilibria for the continuation game with belief $\left\{\mathbf{b}_{2}, \mathbf{b}_{3}\right\}$ at time $t=1$. One equilibrium is for the pooling accepting choice rule in which both buyer types offer 42 . In this case, both buyer types will accept the offer in equilibrium. The other is for the separating choice rule in which buyer $\mathbf{b}_{2}$ offers 50 but buyer $\mathbf{b}_{3}$ offers -1 . In this case, $\mathbf{b}_{2}$ 's offer will be accepted at time $t=2$. $\mathbf{b}_{3}$ 's offer will be rejected and the seller will offer 50 after updating its belief to $\left\{\mathbf{b}_{3}\right\}$.

Now we consider the seller's equilibrium offers with the initial belief at time $t=0$. Seller s can apply the following different reject update rules:

1. If the buyer rejects the seller s's offer, seller $\mathbf{s}$ will not change its belief, i.e., seller $\mathbf{s}$ is offering $\varpi$ by convention. Under this reject update rule, any buyer type's acceptance price depends on the negotiation outcome in the continuation game $\Gamma\left(1,\left\{\mathbf{b}_{1}, \mathbf{b}_{2}, \mathbf{b}_{3}\right\}\right)$, which has three sequential equilibria. In the first sequential equilibrium where all buyer types adopt the pooling accepting choice rule at time $t=1$, all buyer types will offer 42 and the seller will accept it at time $t=2$. If seller $\mathbf{s}$ offers $\varpi$, its utility is $(42-10) 0.8^{2}=20.48$. 
Buyer types' acceptance prices are $(42) \leftarrow \mathbf{b}_{1}=65.2$ for $\mathbf{b}_{1},(42) \leftarrow \mathbf{b}_{2}=49.2$ for $\mathbf{b}_{2}$, $(42)_{\leftarrow \mathbf{b}_{3}}=45.2$ for $\mathbf{b}_{3}$. If seller $\mathbf{s}$ offers 65.2 , buyer type $\mathbf{b}_{1}$ will accept it but buyer types $\mathbf{b}_{2}$ and $\mathbf{b}_{3} 2$ will reject it and follow the first sequential equilibrium. Thus, seller $\mathbf{s}$ 's expected utility while offering 65.2 is $0.25 \cdot(65.2-10) \cdot 0.8+0.75 \cdot(42-10) 0.8^{2}=$ 26.40. In the same way, we can find that the seller achieves an expected utility of 28.64 while offering 49.2 and achieves an expected utility of 28.16 while offering 45.2 . Thus, the seller's optimal offer is 49.2 . However, buyer types $\mathbf{b}_{1}$ and $\mathbf{b}_{2}$ will accept the offer, which is in conflict with the reject update rule in which all buyer types will reject the offer. Therefore, there is no sequential equilibrium for the first sequential equilibrium for the continuation game $\Gamma\left(1,\left\{\mathbf{b}_{1}, \mathbf{b}_{2}, \mathbf{b}_{3}\right\}\right)$. In the same way, we can see that there is no sequential equilibrium for the other two sequential equilibria for the continuation game $\Gamma\left(1,\left\{\mathbf{b}_{1}, \mathbf{b}_{2}, \mathbf{b}_{3}\right\}\right)$.

2. If the buyer rejects the seller $\mathbf{s}$ 's offer, seller $\mathbf{s}$ updates its belief to $\left\{\mathbf{b}_{3}\right\}$, i.e., seller $\mathbf{s}$ is making an offer acceptable to $\mathbf{b}_{1}, \mathbf{b}_{2}$. There is only one sequential equilibrium for the continuation game $\Gamma\left(1,\left\{\mathbf{b}_{3}\right\}\right)$ in which type $\mathbf{b}_{3}$ offers $x_{\mathbf{b}_{3}}^{*}(1)=42.0$. It is easy to see that seller $\mathbf{s}$ gets a utility of $(42-10) 0.8^{2}=20.48$ if it offers $\varpi$ at time 0 . Buyer types' acceptance prices are $(42) \leftarrow \mathbf{b}_{1}=65.2$ for $\mathbf{b}_{1},(42) \leftarrow \mathbf{b}_{2}=49.2$ for $\mathbf{b}_{2},(42) \leftarrow \mathbf{b}_{3}=45.2$ for $\mathbf{b}_{3}$. Seller s's optimal offer for this reject update rule is 49.2 . We can easily see that no buyer type has an incentive to deviate: (1) By rejecting the offer 49.2, buyer type $\mathbf{b}_{1}$ can gain a utility of $(100-42) 0.6^{2}=20.88$, which is lower than the utility $(100-49.2) 0.6=30.48$ when it accepts the offer. (2) Buyer type $\mathbf{b}_{2}$ gains a utility of $(60-49.2) 0.6=6.48$ by accepting the offer 49.2 , which is not lower than its utility $(60-42) 0.6^{2}=6.48$ when it rejects the offer. (3) Buyer type $\mathbf{b}_{3}$ gains a utility of $(50-49.2) 0.6=0.48$ by accepting the offer 49.2 , which is lower than its utility $(50-42) 0.6^{2}=2.88$ when it rejects the offer.

3. If the buyer rejects the seller $\mathbf{s}$ 's offer, seller $\mathbf{s}$ updates its belief to $\left\{\mathbf{b}_{2}, \mathbf{b}_{3}\right\}$, i.e., seller $\mathbf{s}$ is making an offer only acceptable to $\mathbf{b}_{1}$. There are two sequential equilibria for the continuation game $\Gamma\left(1,\left\{\mathbf{b}_{2}, \mathbf{b}_{3}\right\}\right)$. For the sequential equilibrium in which both buyer types offer an acceptable price 42 , the seller's optimal offer is 49.2 such that $\mathbf{b}_{1}$ will accept it and $\mathbf{b}_{2}$ and $\mathbf{b}_{3}$ will reject it. However, we could not find an offer which satisfies all equilibrium existence conditions given the other sequential equilibrium for the continuation game $\Gamma\left(1,\left\{\mathbf{b}_{2}, \mathbf{b}_{3}\right\}\right)$.

4. Finally, the seller can make an offer that will be accepted by all buyer types, i.e., seller $\mathbf{s}$ updates its belief to $\left\{\mathbf{b}_{1}\right\}$ if buyer rejects the seller s's offer. The acceptance prices for buyer types $\mathbf{b}_{1}, \mathbf{b}_{2}$, and $\mathbf{b}_{3}$ are 89.2, 60.0, and 50.0, respectively. Seller $\mathbf{s}$ 's optimal offer for this reject update rule is 89.2. Buyer types $\mathbf{b}_{2}$ and $\mathbf{b}_{3}$ will reject the seller's optimal offer, which is in conflicting with the reject update rule. Therefore, there is no sequential equilibrium for this reject update rule.

Therefore, there are two sequential equilibria for the bargaining game. In the first equilibrium, seller $\mathbf{s}$ will offer 49.2 at time $t=0$. If the buyer is of type $\mathbf{b}_{1}$ or $\mathbf{b}_{2}$, it will accept the offer and they make an agreement at time $t=1$. If the buyer is of type $\mathbf{b}_{3}$, it will reject the offer and make a counter offer 42.0 at time $t=1$. When seller $\mathbf{s}$ receives offer 42.0 , it will update its belief to $\left\{\mathbf{b}_{3}\right\}$ and it will accept the offer at time $t=2$. In the second equilibrium, seller $\mathbf{s}$ will also offer 49.2 at time $t=0$. If the buyer is of type $\mathbf{b}_{1}$, it will accept the offer and they make an agreement at time $t=1$. If the buyer is of type $\mathbf{b}_{2}$ or $\mathbf{b}_{3}$, it will reject the offer and make a counter offer 42.0 at time $t=1$. When seller $\mathbf{s}$ receives offer 42.0 , it will update its belief to $\left\{\mathbf{b}_{2}, \mathbf{b}_{3}\right\}$ and it will accept the offer at time $t=2$. 


\section{Applications of the approach}

Our approach can be used to compute pure strategy sequential equilibria in other games with continuous strategy space. This section briefly discusses two potential application of our proposed approach.

\subsection{Bilateral negotiation with uncertain discount factor}

This paper considers one-sided uncertainty regarding reserve prices and we assume complete knowledge about agents' discount factors. In other cases, one agent may have incomplete information about the other agent's discount factors. For example, we can assume that the buyer $\mathbf{b}$ can be of finitely many types $\left\{\mathbf{b}_{1}, \ldots, \mathbf{b}_{n}\right\}$ in which buyer $\mathbf{b}_{i}$ has a discount factor $\delta_{\mathbf{b}}^{i}$. The initial belief of $\mathbf{s}$ on $\mathbf{b}$ is $\mu(0)=\left\langle\Delta_{\mathbf{b}}^{0}, P_{\mathbf{b}}^{0}\right\rangle$ where $\Delta_{\mathbf{b}}^{0}=\left\{\mathbf{b}_{1}, \ldots, \mathbf{b}_{n}\right\}$ and $P_{\mathbf{b}}^{0}=\left\{\omega_{\mathbf{b}_{1}}^{0}, \ldots, \omega_{\mathbf{b}_{n}}^{0}\right\}$ such that $\sum_{i} \omega_{\mathbf{b}_{i}}^{0}=1 . \omega_{\mathbf{b}_{i}}^{0}$ is the prior probability that $\mathbf{b}$ is of type $\mathbf{b}_{i}$. Let $x_{\mathbf{b}_{i}}^{*}(t)$ be any agent optimal offer at time $t$ when $\mathbf{b}$ is of type $\mathbf{b}_{i}$ in this case. It follows that $x_{\mathbf{b}_{i}}^{*}(t) \leq x_{\mathbf{b}_{j}}^{*}(t)$ if $\delta_{\mathbf{b}}^{i}>\delta_{\mathbf{b}}^{j}$.

Lemma $13 x_{\mathbf{b}_{i}}^{*}(t) \leq x_{\mathbf{b}_{j}}^{*}(t)$ if $\delta_{\mathbf{b}}^{i}>\delta_{\mathbf{b}^{j}}^{j}$.

Proof Case $1(\iota(T)=\mathbf{s})$. It follows that $x_{\mathbf{b}_{i}}^{*}(T-1)=x_{\mathbf{b}_{j}}^{*}(T-1)=\operatorname{RP}_{\mathbf{s}}$. Then $x_{\mathbf{b}_{i}}^{*}(T-2)=$ $\mathrm{RP}_{\mathbf{b}}-\delta_{\mathbf{b}}^{i}\left(\mathrm{RP}_{\mathbf{b}}-x_{\mathbf{b}_{i}}^{*}(T-1)\right)<x_{\mathbf{b}_{j}}^{*}(T-2)=\mathrm{RP}_{\mathbf{b}}-\delta_{\mathbf{b}^{j}}^{j}\left(\mathrm{RP}_{\mathbf{b}}-x_{\mathbf{b}_{i}}^{*}(T-1)\right)$. Similarly, we have $x_{\mathbf{b}_{i}}^{*}(T-3)=\mathrm{RP}_{\mathbf{s}}\left(1-\delta_{\mathbf{s}}\right)+\delta_{\mathbf{s}} x_{\mathbf{b}_{i}}^{*}(T-2)$ and $x_{\mathbf{b}_{j}}^{*}(T-3)=\operatorname{RP}_{\mathbf{s}}\left(1-\delta_{\mathbf{s}}\right)+\delta_{\mathbf{s}} x_{\mathbf{b}_{j}}^{*}(T-2)$. Thus we have $x_{\mathbf{b}_{i}}^{*}(T-3)<x_{\mathbf{b}_{j}}^{*}(T-3)$. Recursively, we have $x_{\mathbf{b}_{i}}^{*}(t)<x_{\mathbf{b}_{j}}^{*}(t)$ for $t<T-3$.

Case $2(\iota(T)=\mathbf{b})$. It follows that $x_{\mathbf{b}_{i}}^{*}(T-1)=x_{\mathbf{b}_{j}}^{*}(T-1)=\mathrm{RP}_{\mathbf{b}}$. Then at time $T-2$, we have $x_{\mathbf{b}_{i}}^{*}(T-2)=\mathrm{RP}_{\mathbf{s}}\left(1-\delta_{\mathbf{s}}\right)+\delta_{\mathbf{s}} x_{\mathbf{b}_{i}}^{*}(T-1)=x_{\mathbf{b}_{j}}^{*}(T-2)$. Thus, $x_{\mathbf{b}_{i}}^{*}(T-2)>x_{\mathbf{b}_{j}}^{*}(T-2)$. As in case 1 , we have $x_{\mathbf{b}_{i}}^{*}(t)<x_{\mathbf{b}_{j}}^{*}(t)$ for $t<T-2$.

In this setting, it is easy to see that the weakest type is the buyer type with the lowest discount factor. Accordingly, if the buyer acts off the equilibrium path, the seller will update its belief to the buyer type with the lowest discount factor following the optimistic conjecture.

One can directly apply our approach to solve the bargaining game with uncertain discount factors. For the bargaining game with uncertain reserve prices, we use two techniques to reduce the number of choice rules and reject update rules that need to be considered. Fortunately, we can still only need to a small number of choice rules and reject update rules for the bargaining game with uncertain discount factors.

First we consider the reject update rule such that the seller will update its belief to $\Delta_{\mathbf{b}}^{\prime} \subseteq \Delta_{\mathbf{b}}$ if the seller's offer is rejected by the buyer where $\Delta_{\mathbf{b}}$ is the seller's belief while making the offer.

Theorem 14 If there is a reject update rule with updated belief $\Delta_{\mathbf{b}}^{\prime} \subseteq \Delta_{\mathbf{b}}$ such that $\delta_{\mathbf{b}}^{i}>\delta_{\mathbf{b}}^{j}$ for $\mathbf{b}_{i} \in \Delta_{\mathbf{b}} \backslash \Delta_{\mathbf{b}}^{\prime}$ and $\mathbf{b}_{j} \in \Delta_{\mathbf{b}}^{\prime}$, agents' strategies are not sequentially rational.

Proof The result can be proved by contradiction. Assume that there is a sequential equilibrium with this reject update rule. Similar to Theorem 4, equilibrium existence conditions requires that $\left(e_{\mathbf{b}_{j}}^{t+1} \mid \Delta_{\mathbf{b}}^{\prime}\right) \leftarrow \mathbf{b}_{j} \leq\left(e_{\mathbf{b}_{j}}^{t+1} \mid \Delta_{\mathbf{b}}^{\prime}\right) \leftarrow \mathbf{b}_{i}$ where $e_{\mathbf{b}_{j}}^{t+1} \mid \Delta_{\mathbf{b}}^{\prime}$ is $\mathbf{b}_{j}$ 's equivalent offer in the continuation game starting from $t+1$ with belief $\Delta_{\mathbf{b}}^{\prime}$. Since $\delta_{\mathbf{b}}^{i}>\delta_{\mathbf{b}}^{j}$, it follows that $\left(e_{\mathbf{b}_{j}}^{t+1} \mid \Delta_{\mathbf{b}}^{\prime}\right)_{\leftarrow \mathbf{b}_{j}}>\left(e_{\mathbf{b}_{j}}^{t+1} \mid \Delta_{\mathbf{b}}^{\prime}\right)_{\leftarrow \mathbf{b}_{i}}$. There is a contradiction. 
Accordingly, we just need to consider no more than $\left|\Delta_{\mathbf{b}}\right|$ reject update rules without sacrificing completeness of our approach. Similarly, we just need to consider a small number of choice rules due to the following theorem.

Theorem 15 Assume that $\mathbf{b}$ behaves in different ways at a continuation game with belief set $\Delta_{\mathbf{b}}$ where $\Delta_{\mathbf{b}}=\Delta_{\mathbf{b}}^{a} \cup \Delta_{\mathbf{b}}^{r}$ at time $t$. If there is a buyer type $\mathbf{b}_{i} \in \Delta_{\mathbf{b}}^{a}$ and a buyer $\mathbf{b}_{j} \in \Delta_{\mathbf{b}}^{r}$ such that $\delta_{\mathbf{b}}^{i}>\delta_{\mathbf{b}}^{j}$, there is no sequential equilibrium for this choice rule.

Proof Similar to Theorem 7, this result can be proved by contradiction. If there is a sequential equilibrium, the following two conditions are satisfied: (1) Buyer type $\mathbf{b}_{i}$ has no incentive to behave as $\mathbf{b}_{j}$, i.e., $\left(e_{\mathbf{s}}^{t+1} \mid \Delta_{\mathbf{b}}^{a}\right)_{\leftarrow \mathbf{s}} \leq \rho\left(\mathbf{b}_{i}, t, E B O\left(\mathbf{b}_{j}, \wp^{r}\right)\right)$; and (2) Buyer type $\mathbf{b}_{j}$ has no incentive to behave as $\mathbf{b}_{i}$, i.e., $\rho\left(\mathbf{b}_{j}, t, E B O\left(\mathbf{b}_{j}, \wp^{r}\right)\right) \leq\left(e_{\mathbf{s}}^{t+1} \mid \Delta_{\mathbf{b}}^{a}\right) \leftarrow \mathbf{s}$. Therefore, equilibrium existence requires that $\rho\left(\mathbf{b}_{j}, t, E B O\left(\mathbf{b}_{j}, \wp^{r}\right)\right) \leq \rho\left(\mathbf{b}_{i}, t, E B O\left(\mathbf{b}_{j}, \wp^{r}\right)\right)$.

Assume that $E B O\left(\mathbf{b}_{j}, \wp^{r}\right)=\left(x, t^{\prime}\right)$ where $T \geq t^{\prime}>t$. From the definition of equivalent offers, we have $\left(\mathrm{RP}_{\mathbf{b}}-\rho\left(\mathbf{b}_{j}, t, E B O\left(\mathbf{b}_{j}, \wp^{r}\right)\right)\right) \cdot\left(\delta_{\mathbf{b}}^{j}\right)^{t+1}=\left(\mathrm{RP}_{\mathbf{b}}-x\right) \cdot \delta_{\mathbf{b}}^{t^{\prime}}$, which can be rewritten as $\rho\left(\mathbf{b}_{j}, t, E B O\left(\mathbf{b}_{j}, \wp^{r}\right)\right)=\mathrm{RP}_{\mathbf{b}}-\left(\mathrm{RP}_{\mathbf{b}}-x\right) \cdot\left(\delta_{\mathbf{b}}^{j}\right)^{t^{\prime}-t-1}$. Similarly, we have $\rho\left(\mathbf{b}_{i}, t, E B O\left(\mathbf{b}_{j}, \wp^{r}\right)\right)=\mathrm{RP}_{\mathbf{b}}-\left(\mathrm{RP}_{\mathbf{b}}-x\right) \cdot\left(\delta_{\mathbf{b}}^{i}\right)^{t^{\prime}-t-1}$. Since $\delta_{\mathbf{b}}^{i}>\delta_{\mathbf{b}}^{j}$, it follows that $\rho\left(\mathbf{b}_{i}, t, E B O\left(\mathbf{b}_{j}, \wp^{r}\right)\right)<\rho\left(\mathbf{b}_{j}, t, E B O\left(\mathbf{b}_{j}, \wp^{r}\right)\right)$ which contradicts with equilibrium existence conditions.

In summary, our approach can be directly applied to solve the bargaining game with onesided uncertain discount factor and we can use similar techniques to reduce computational cost.

\subsection{Bilateral multi-issue negotiation with uncertain weights}

Another potential application of our approach is bilateral multi-issue negotiation, which is more complex and challenging than a single-issue negotiation since agents need to make tradeoffs between multiple issues. The problem of bargaining efficiently over multiple issues with complete information has been addressed in [15-17]. Agents' equilibrium strategies can be easily computed by extending the backward induction method in Sect. 2. Specifically, the acting agent $\iota(t)$ at time $t$ chooses its best offer (consisting of values for each negotiation issue) that is acceptable to the other agent. In presence of incomplete information, it is common to compute agents' sequential equilibrium strategies. There are different sources of uncertainty. For uncertainty about agents' reserve prices, discount factors or negotiation deadlines, the calculation of sequential equilibria in a multi-issue negotiation game is the same as that in a single issue negotiation game. The only new source of uncertainty introduced by having multiple issues is the uncertainty regarding the weights of different issues.

In multi-issue negotiation, two agents are negotiating over multiple issues $1, \ldots, l$. For each issue $i$, let $\mathrm{RP}_{\mathbf{a}}^{i}$ be agent $\mathbf{a}$ 's reserve price for the issue. A negotiation outcome can be represented as $o=\left\langle o_{1}, \ldots, o_{l}\right\rangle$. An agent a's utility of a negotiation outcome $o$ is defined as $U_{\mathbf{a}}(o)=\sum_{1 \leq i \leq l} w_{\mathbf{a}}^{i} U_{\mathbf{a}}\left(o_{i}\right)$ where $U_{\mathbf{a}}\left(o_{i}\right)$ is a's utility given the negotiation outcome $o_{i}$ for issue $i$, which is the same as the utility function in single issue negotiation (see Sect. 2). In the cumulative utility function, $w_{\mathbf{a}}^{i}$ is agent a's weight for issue $i$. Let $w_{\mathbf{a}}=\left\langle w_{\mathbf{a}}^{1}, \ldots, w_{\mathbf{a}}^{l}\right\rangle$ be agent a's weight vector. We consider the one-sided uncertainty about the buyer's weights of the issues and all other parameters are complete information. There are $n$ possible weight vectors $\left\{w_{\mathbf{b}_{1}}, \ldots, w_{\mathbf{b}_{n}}\right\}$ for the buyer and the probability of the buyer being the type $w_{\mathbf{b}_{i}}$ is $\omega_{\mathbf{b}_{i}}$. The probability distribution is common knowledge.

Fatima et al. $[16,17]$ present an algorithm to produce equilibrium strategies in multi-issue bargaining with uncertain weights but the strategies found by their algorithm are not neces- 


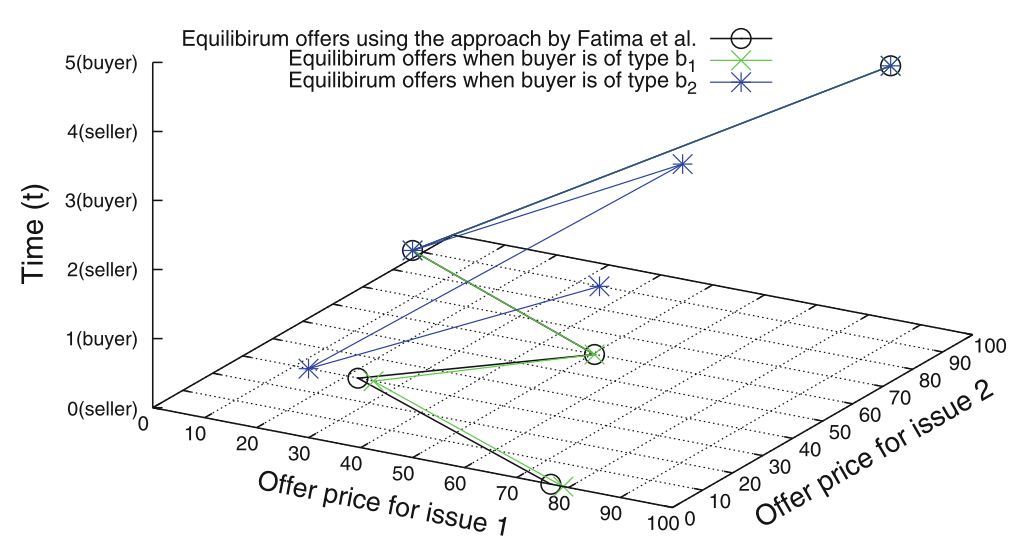

Fig. 3 Failure of the approach in $[16,17]$ with $T=5, \iota(0)=\mathbf{s}, \mathrm{RP}_{\mathrm{s}}^{1}=\mathrm{RP}_{\mathrm{s}}^{2}=0, \mathrm{RP}_{\mathrm{b}}^{1}=\mathrm{RP}_{\mathrm{b}}^{2}=90, \delta_{\mathrm{s}}=0.5$, $\delta_{\mathrm{b}}=0.8, w_{\mathrm{s}}=\langle 0.9,0.1\rangle,\left\{w_{\mathrm{b}_{1}}=\langle 0.4,0.6\rangle, w_{\mathrm{b}_{2}}=\langle 0.9,0.1\rangle\right\}, \omega_{\mathrm{b}_{1}}=0.9$, and $\omega_{\mathrm{b}_{2}}=0.1$; agents' offers in complete information settings were also showed

sarily sequentially rational given the designed system of beliefs as we discussed previously. ${ }^{6}$ Here we show a simple example (see Fig. 3) where buyer $\mathbf{b}$ and seller $\mathbf{s}$ are negotiating over two issues 1 and 2 with the following parameters: $T=5, \iota(0)=\mathbf{s}, \mathrm{RP}_{\mathbf{s}}^{1}=\mathrm{RP}_{\mathbf{s}}^{2}=0, \mathrm{RP}_{\mathbf{b}}^{1}=$ $\mathrm{RP}_{\mathbf{b}}^{2}=90, \delta_{\mathbf{s}}=0.5, \delta_{\mathbf{b}}=0.8, w_{\mathbf{s}}=\langle 0.9,0.1\rangle$. There are 2 possible weight vectors $\left\{w_{\mathbf{b}_{1}}=\langle 0.4,0.6\rangle, w_{\mathbf{b}_{2}}=\langle 0.9,0.1\rangle\right\}$ for the buyer and the probability of the buyer being the type $w_{\mathbf{b}_{1}}$ and $w_{\mathbf{b}_{2}}$ are $\omega_{\mathbf{b}_{1}}=0.9$ and $\omega_{\mathbf{b}_{2}}=0.1$, respectively. Assume that seller $\mathbf{s}$ is the agent $a$ in the formulation in $[16,17]$. At time $t=4$, function TRADEOFFA1 in [16,17] returns the seller's best offer, which are the buyer's reserve prices for both issues $\langle 90,90\rangle$. This optimal offer gives the seller a utility of $0.9 \cdot(90-0) \cdot 0.5^{5}+0.1 \cdot(90-0) \cdot 0.5^{5}=2.8125$. At time $t=3$, both buyer types' optimal offer that is acceptable to the seller is $\langle 50,0\rangle$, which is returned by function TRADEOFFB1 in $[16,17]$. The seller is indifferent between accepting the offer $\langle 50,0\rangle$ or making a counter-offer $\langle 90,90\rangle$ at time $t=4$. At time $t=2$, seller's optimal offer that is acceptable to buyer type $\mathbf{b}_{1}$ is $\langle 85,0\rangle$ which can give seller an expected utility of 8.8875 . Seller's optimal offer that is acceptable to buyer type $\mathbf{b}_{2}$ is $\langle 50,90\rangle$ which can give seller an expected utility of 3.20625. Function TRADEOFFA1 returns seller's optimal offer at time $t=2$, which is $\langle 85,0\rangle$. In the same way, we can compute that both buyer types' optimal offer at time $t=1$ is $\langle 39.5,0\rangle$. Similarly, we can compute that seller's optimal offer at time $t=0$ is $\langle 76.6,0\rangle$, which is only acceptable to the buyer type $\mathbf{b}_{1}$. That is, if the buyer rejects the offer, the seller will update is belief to $\left\{\mathbf{b}_{2}\right\}$. However, buyer type $\mathbf{b}_{1}$ has an incentive to reject the offer and to make buyer type $\mathbf{b}_{2}$ complete information offer $\langle 30,0\rangle$ which can give $\mathbf{b}_{1}$ a higher utility than accepting seller's offer $\langle 76.6,0\rangle$. Thus, the equilibrium computed using the approach in $[16,17]$ is not sequential rational.

We can apply our approach to solve the multi-issue bargaining game with uncertain issue weights in Fig. 3. At time $t=0$, we need to try different reject update rules and for each reject update rule, we first compute the sequential equilibria for its continuation game starting from time $t=1$. To compute all the sequential equilibria for a continuation game starting from time $t=1$, we need to consider different choice rules. While computing agents' equilibrium

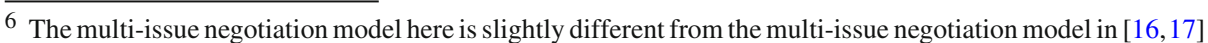
where two negotiation agents are splitting pies and the size of each pie shrinks over time due to the discount factors. In contrast, the utility of each agent shrinks over time. Our formulation of multi-issue negotiation has been widely used, e.g., [13,15], just to name a few.
} 
offers, one important optimization problem is computing one buyer type's (or a seller's) optimal offer which can give the seller (or a buyer type) certain utility. For instance, seller's expected utility is $y>0$ and buyer type $\mathbf{b}_{j}$ with weights $w_{\mathbf{b}_{j}}=\left\langle w_{\mathbf{b}_{j}}^{1}, \ldots, w_{\mathbf{b}_{j}}^{l}\right\rangle$ is finding a package offer $x=\left\langle x^{1}, \ldots, x^{l}\right\rangle$ at time $t$ to maximize its utility. The optimization problem can be formulated as

$$
\begin{gathered}
\text { maximise } \sum_{1 \leq i \leq l} w_{\mathbf{b}_{j}}^{i} \cdot\left(\mathrm{RP}_{\mathbf{b}}^{i}-x^{i}\right) \cdot\left(\delta_{\mathbf{b}}\right)^{t+1} \\
\text { such that } \sum_{1 \leq i \leq l} w_{\mathbf{s}}^{i} \cdot\left(x^{i}-\mathrm{RP}_{\mathbf{s}}^{i}\right) \cdot\left(\delta_{\mathbf{s}}\right)^{t+1} \geq y \\
\operatorname{RP}_{\mathbf{b}}^{i} \leq x^{i} \leq \mathrm{RP}_{\mathbf{b}}^{i} \text { for } 1 \leq i \leq l
\end{gathered}
$$

As implied in $[16,17]$, the optimal solution can be generated using a greedy approach by considering $w_{\mathbf{s}}^{i} / w_{\mathbf{b}_{j}}^{i}$ for $1 \leq i \leq l$ where $w_{\mathbf{b}_{j}}^{i} / w_{\mathbf{s}}^{i}$ is the utility that $\mathbf{b}_{j}$ needs to give up in order increase s's utility by $\left(\delta_{\mathbf{b}}\right)^{t+1} /\left(\delta_{\mathbf{s}}\right)^{t+1}$. Thus, $\mathbf{b}_{j}$ begins by making concessions to seller $\mathbf{s}$ on the issue with the lowest $w_{\mathbf{b}_{j}}^{i} / w_{\mathbf{s}}^{i}$ value. Accordingly, the complexity of solving the optimization problem is polynomial.

The weakness of different buyer types at any continuation game can be computed by solving the bargaining game with complete information about weights of negotiation issues. Recall that the weakest type gives the seller the highest utility in the complete information bargaining setting. Rather than only considering a small set of reject update rules and choice rules, here we may need to consider all reject update rules and choice rules since the two computation reduction techniques are not necessarily valid here. However, this only increases the number of computations and does not affect the applicability of our approach. One sequential equilibrium for the multi-issue bargaining game in Fig. 3 is that at time $t=0$, seller $\mathbf{s}$ makes an offer $\langle 69,0\rangle$ that is only acceptable to buyer type $\mathbf{b}_{1}$. It follows that (1) offer $\langle 69,0\rangle$ is the seller's optimal offer for this reject update rule; (2) buyer type $\mathbf{b}_{1}$ gains a utility of 49.92 and it has no incentive to reject the offer; and (3) by rejecting the offer, buyer type $\mathbf{b}_{2}$ will gain a utility of 40.32 , which is higher than the utility 22.32 by accepting the offer $\langle 69,0\rangle$.

\section{Existing solutions in literature}

Computing agents' equilibrium strategies of an extensive-form game with imperfect information is well known to be hard and classic game theory does not provide any general approaches to find sequential equilibria. While there has been long standing literature in solving bargaining games with uncertainty since Rubinstein's path-breaking work [32], there is no existing approach that can be applied to compute pure strategy sequential equilibria for the problem studied in this paper. An interested reader can find a more detailed survey on bargaining with uncertainty in [7].

Computer science researchers have proposed a number of algorithms for computing Nash equilibria (e.g., [22,23,26]) or sequential equilibria (e.g., [20,29]). However, these algorithms are not applicable in solving bargaining games since they only consider finite strategy space rather than continuous strategy space considered in this paper. Due to the same reason, enumeration based methods (e.g., [30]) cannot be applied to our bargaining problem as well.

The microeconomics literature provides a number of results for some specific bargaining problems with uncertainty. For instance, [9-11,32] considered bilateral infinite horizon bargaining with uncertainty and those approaches cannot be applied here due to the unrealistic 
infinite horizon assumption. Sandholm and Vulkan [35] analyze agents' strategic behavior in a slight variation of the war-of-attrition game where the surplus can be divided. They consider a finite horizon alternating-offer bilateral bargaining game where agents have uncertain deadlines, time is continuous, and there are not discount factors. In contrast, Gatti et al. [19] relaxed the infinite horizon deadlines and provided an algorithm to compute agents' equilibrium strategies in bilateral bargaining with one-sided uncertain deadlines. They proved that agent types would adopt the same strategy at any time point before their deadlines, which may not be true in our case with uncertain reserve prices. Therefore, their approach cannot be applied to our case. An et al. [3] only considered two-type uncertainty about reserve prices and their approach cannot be directly extended to handle multiple types. They considered the situation that different buyer types can make the same/different offers. However, they assume that the seller always makes an offer that will be accepted by all the buyer types. Therefore, in presence of multi-type uncertainty, the algorithm in An et al. [3] is no longer complete. The algorithm presented in [8] solves bargaining situations with any form of one-sided uncertainty by reducing the bargaining game to a finite auxiliary game and then solving this by using a variation of the Lemke algorithm (exploiting a specific lexicographic perturbation able to find a sequential equilibrium [29]). The auxiliary game is exponential in the number of types and it can be solved only with very few types $(<5)$ and short deadlines $(<10)$. As shown experimentally, our algorithm can compute all sequential equilibria for games with 9 types and 14 negotiation rounds within a reasonable time. In addition, the algorithm suffers numerical stability since the matrix associated with the tableau used by the Lemke algorithm is badly conditioned and, as a result, the algorithm may not terminate, unless arbitrary precision is used (but, with arbitrary precision, the performance of the algorithm drastically worsens). Another drawback of this algorithm is that it returns a single equilibrium not allowing the equilibrium selection. Thus, the algorithm may return an equilibrium in mixed strategies, even if there is a more satisfactory equilibrium in pure strategies.

The only known general approach that be potentially applied to our bargaining problem to compute pure strategy equilibria is the backward induction approach by Fatima et al. [16,17]. They studied the alternating-offers protocol with multiple negotiation issues and uncertainty over the weights of the issues. They proposed an algorithm based on backward induction to compute sequential equilibria. Note that as in this paper, Fatima et al. [16,17] also focus on pure strategy equilibria. Basically, their algorithm searches in the space of the strategies exploiting the backward induction from the last possible deadline to $t=0$ with agents' initial beliefs, and, once the optimal strategies at time point $t=0$ have been found, the system of beliefs is designed to be consistent with them. Gatti et al. [19] have shown that unfortunately an equilibrium returned by their algorithm is not necessary a sequential equilibrium in pure strategies for bilateral bargaining with uncertain deadlines.

We show a single-issue negotiation example with uncertain reserve prices where the algorithm in $[16,17]$ fails in the bargaining problem studied in this paper (see Fig. 4 for agents' equilibrium offers computed by their algorithm and agents' equilibrium offers in complete information settings). Consider the following scenario: $T=5, \iota(0)=\mathbf{s}, \mathrm{RP}_{\mathbf{s}}=10, \mathrm{RP}_{1}=$ $90, \mathrm{RP}_{2}=70, \omega_{\mathbf{b}_{1}}^{0}=0.8, \omega_{\mathbf{b}_{2}}^{0}=0.2, \delta_{\mathbf{s}}=0.7$, and $\delta_{\mathbf{b}}=0.8$. Let $x_{\mathbf{b}_{i}}^{*}(t)$ be any agent optimal offer at time $t$ when buyer $\mathbf{b}$ is of type $\mathbf{b}_{i}$ in the complete information setting. Let $x^{f}(t)$ be any agent optimal offer at time $t$ computed by the algorithm in [16,17]. Agents' equilibrium offers are computed with the initial belief. At time $t=4$, seller $\mathbf{s}$ can offer either $x_{\mathbf{b}_{1}}^{*}(4)=\mathrm{RP}_{1}=90$ which gives the seller a utility of $0.8 \cdot(90-10) \cdot 0.7^{5}=10.75648$ or $x_{\mathbf{b}_{2}}^{*}(4)=\mathrm{RP}_{2}=70$ which give the seller a utility of $(70-10) \cdot 0.7^{5}=10.0842$. Therefore, the seller's optimal offer at time $t=4$ is $x^{f}(4)=90$. Then both buyer types' optimal offer at time $t=3$ is 


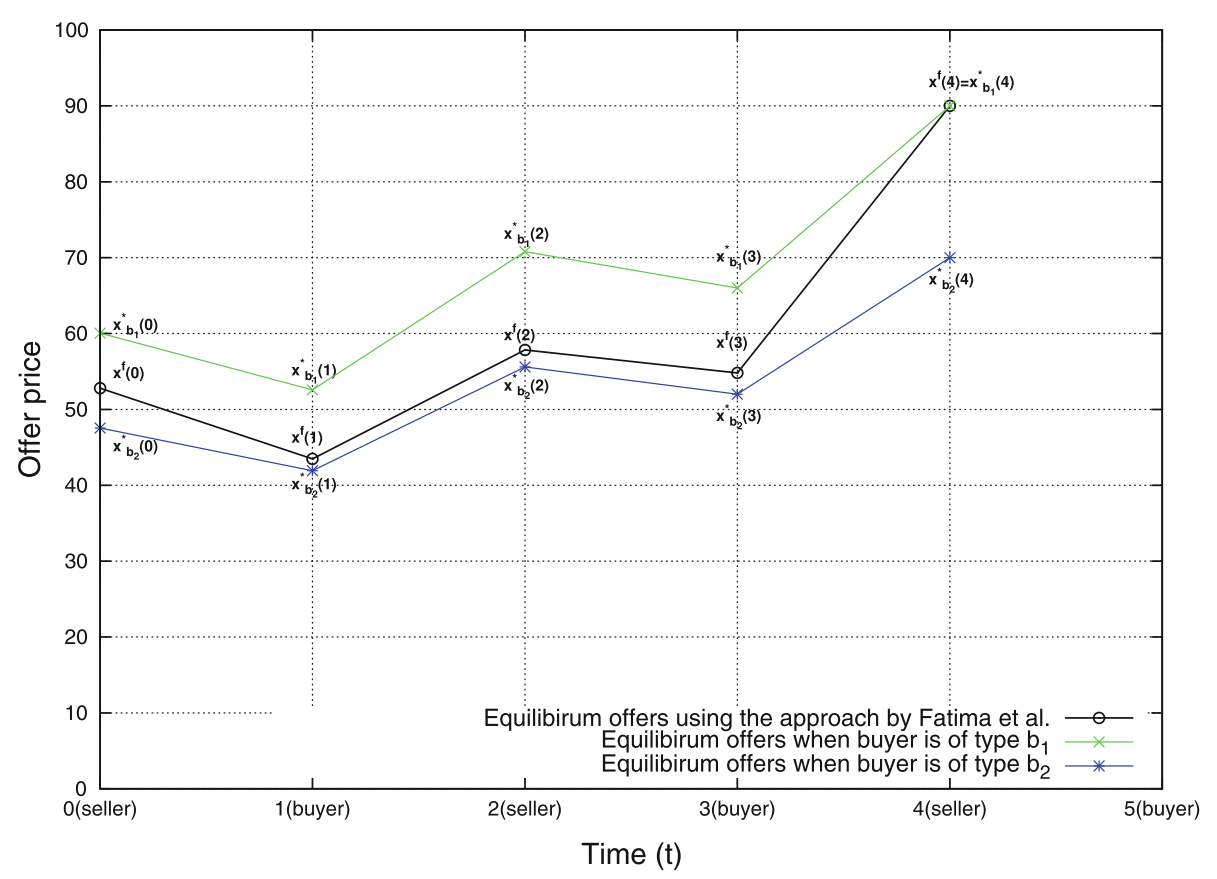

Fig. 4 Failure of the approach in $[16,17]$ with $T=5, \iota(0)=\mathrm{s}, \mathrm{RP}_{\mathrm{s}}=10, \mathrm{RP}_{1}=90, \mathrm{RP}_{2}=70, \omega_{\mathrm{b}_{1}}^{0}=0.8$, $\omega_{\mathrm{b}_{2}}^{0}=0.2, \delta_{\mathrm{s}}=0.7$, and $\delta_{\mathrm{b}}=0.8$; agents' offers in complete information settings were also showed

$x^{f}(3)$ such that $\left(x^{f}(3)-10\right) \cdot 0.7^{4}=10.75648$, i.e., $x^{f}(3)=54.8$. At time $t=2$, seller $\mathbf{s}$ can offer either $(54.8) \leftarrow \mathbf{b}_{1}=61.84$ or $(54.8)_{\leftarrow \mathbf{b}_{2}}=57.84$. According to [16,17], offering price 57.84 will be accepted by both buyer types and thus the seller can gain a utility of $(57.84-10) \cdot 0.7^{3}=16.40912$. In contrast, offering price 61.84 will only be accepted by buyer type $\mathbf{b}_{1}$ and the seller's equilibrium offer at time $t=4$ will be accepted. Thus, offering price 61.84 will give the seller a utility of $0.8 \cdot(61.84-10) \cdot 0.7^{3}+0.2 \cdot(74-10) \cdot 0.7^{5}=$ 16.376192. Thus the optimal offer of the seller at time $t=2$ is $x^{f}(2)=57.84$. Then both buyer types' optimal offer at time $t=1$ is $x^{f}(1)=(57.84)_{\leftarrow \mathbf{s}}=43.488$. The seller at time $t=0$ can offer either $(43.488) \leftarrow \mathbf{b}_{1}=52.7904$ or $(43.488)_{\leftarrow \mathbf{b}_{2}}=48.7904$ and its optimal offer is $x^{f}(0)=52.7904$ which will only be accepted by buyer type $\mathbf{b}_{1}$. According to [16,17], buyer type $\mathbf{b}_{1}$ will accept the offer 52.7904 at time $t=0$ since the optimal offer 52.7904 is $\mathbf{b}_{1}$ 's backward propagated value of its 43.488 at time $t=1$. Accordingly, the seller will update its belief as follows: if its optimal offer 52.7904 is rejected, it will update its belief to $\left\{\mathbf{b}_{2}\right\}$.

However, the above strategy profile is not in sequential equilibrium since buyer $\mathbf{b}_{1}$ has an incentive to reject the seller's equilibrium offer at time $t=1$ (also see Fig. 4). If buyer type $\mathbf{b}_{1}$ rejects the offer 52.7904 at time $t=1$ and makes a counter offer 41.92, the seller will accept it since 41.92 is buyer's equilibrium offer when the buyer is of type $\mathbf{b}_{2}$. By doing so, buyer $\mathbf{b}_{1}$ gains a utility of $(90-41.92) \cdot 0.8^{2}=30.7712$ which is higher than its utility $(90-52.7904) \cdot 0.8=29.76768$ when it accepts the seller's equilibrium offer 52.7904. As pointed out in [19], the reason behind the failure of $[16,17]$ in producing equilibrium strategies for some settings of parameters is that in each step of backward induction they limit the search to the space of the strategies, but they do not verify the existence of a consistent 
system of beliefs such that the found strategy is sequentially rational. In other words, they break the circularity of strategies and belief systems. In the above example, they decide the acceptance price of buyer type $\mathbf{b}_{1}$ with the initial belief and ignore the effect of the seller's belief update rule. As a result, once their algorithm has produced the agents' strategies at $t=0$ and has designed the system of beliefs consistent with them, the strategies may not be sequentially rational given the designed system of beliefs.

In the above work in strategic bargaining theory, agents are assumed to be fully rational. Due to the difficulties of studying strategic bargaining games, many authors have proposed to relax the assumption of full rationality. More precisely, agents with bounded rationality are constrained to play heuristic strategies. For example, to build more flexible and sophisticated negotiation agents, Faratin etal. [14] devised a negotiation model that defines a range of negotiation decision functions for generating (counter-)proposals based on time, resource, and behaviors of negotiators. Heuristic based approaches have received a lot of attention in the recent years and have been applied to many practical applications such as streaming processing systems [2], multi-resource negotiation [5], and cloud computing [4]. The major disadvantage of this line of research is choosing heuristics in an ad hoc way and thus agents' strategies are usually neither optimal nor in equilibrium.

\section{Conclusions}

Studying rational agents' strategic behavior is currently one of the most interesting issues in the field of automated negotiation. However, the bargaining theory literature lacks of general solutions for bargaining games with the presence of deadlines and incomplete information. In this paper we go beyond state of the art by providing an algorithm that can find all sequential equilibria in incomplete information bargaining games with deadline constraints. Specifically, this paper analyzes agents' rational strategic behavior in alternating-offers bilateral bargaining with deadline constraints and one-sided uncertainty on reserve prices. Our approach computes sequential equilibrium employing backward induction. To guarantee the completeness of our approach, we enumerate all choice rules and belief reject update rules. To guarantee the soundness of our approach, we construct equilibrium existence conditions along the backward induction process. Our approach can also be applied to other uncertainty settings, e.g., bilateral multi-issue negotiation with uncertain weight functions $[16,17]$, and bilateral bargaining with uncertain discount factors.

Our study shows that there exists at least one sequential equilibrium in more than $99.7 \%$ of scenarios we have tried in which there are deadline constraints and incomplete information. There are two future research directions for this equilibrium nonexistence problem. On one hand, we can develop algorithm for finding mixed equilibrium strategies for bargaining scenarios in which there is no pure strategy equilibrium. On the other hand, we can slightly modify the alternating-offers protocol that would allow the existence of the equilibrium in pure strategies, e.g., the introduction of agents' strategic delay option [12].

While this paper only considers one-sided uncertainty, we think our approach can be extended to handle two-sided uncertainty. Assume that the buyer is also uncertain about the seller's reserve price. When it is the buyer's turn to make an offer, rather than considering whether a buyer type's offer will be accepted be the seller, we need to consider the set of seller types that will accept the buyer's offer. That is, we need to combine choice rules and reject update rules. Similar to the buyer types, all seller types need to not only consider different reject update rules but also different choice rules. In addition to two-sided uncertainty 
regarding reserve prices, our algorithm can also potentially handle two-sided uncertainty about discount factors.

One major motivation of the study of bargaining theory is designing successful bargaining agents in practical markets where there are more uncertainty and more agents. Although constraints, complexity, and uncertainty make it impractical to develop optimal negotiation strategies, our analysis can still give us some insights into the bargaining problems. Consider that a buyer is acquiring multiple resources in a dynamic market with multiple sellers. We can first use our approach to generate the strategy for each single seller and then use heuristics for all sellers to combine the set of strategies to generate the overall negotiation strategy.

There are several other natural directions suggested by our research. The first one concerns the extension of our game theoretic analysis to incomplete information bargaining games with multiple agents (including both buyers and sellers). Second, we have seen from experimental results that there are more than one pure strategy sequential equilibrium in some scenarios. It would be useful to design coordination mechanisms for choosing certain equilibrium strategies for agents to play. In addition, characterizing bargaining games with no sequential equilibrium is also on the agenda. Finally, our experiments about the performance of equilibrium strategies thus far have focused on scenarios ranging from low to moderate complexity, but we wish to investigate much larger problems where there are longer deadlines and more buyer types. We have done some initial work in exploring some static heuristic strategies and their performance in relationship to the sequential equilibria strategies produced by our algorithm [1]. For this initial set of strategies, we demonstrated that in fact as expected the heuristic strategies were significantly worse against out algorithm. However, in order to be more firm about this conclusion we want to explore a wide range of heuristic strategies, especially one that are more dynamic.

Acknowledgments The authors would like to thank the Editors and the anonymous reviewers for their suggestions for improving the accuracy and readability of the paper.

\section{References}

1. An, B. (2011). Automated negotiation for complex multi-agent resource allocation. $\mathrm{PhD}$ thesis, University of Massachusetts Amherst.

2. An, B., Douglis, F., \& Ye, F. (2008). Heuristics for negotiation schedules in multi-plan optimization. In Proceedings of the international joint conference on autonomous agents and multi-agent systems, pp. 551-558.

3. An, B., Gatti, N., \& Lesser, V. (2009). Bilateral bargaining with one-sided two-type uncertainty. In Proceedings of the IEEE/WIC/ACM international conference on intelligent agent technology (IAT), pp. 403-410.

4. An, B., Lesser, V., Irwin, D., \& Zink, M. (2010). Automated negotiation with decommitment for dynamic resource allocation in cloud computing. In Proceedings of the international joint conference on autonomous agents and multi-agent systems, pp. 981-988.

5. An, B., Lesser, V., \& Sim, K. M. (2011). Strategic agents for multi-resource negotiation. Journal of Autonomous Agents and Multi-Agent Systems, 23, 114-153.

6. Aumann, R. (1985). What is game theory trying to accomplish? In Frontiers of economics (pp. 909-924). Oxford: Basil Blackwell.

7. Ausubel, L. M., Cramton, P. C., \& Deneckere, R. J. (2002). Bargaining with incomplete information. In Handbook of game theory (Vol. 3, pp. 1897-1945). Amsterdam: Elsevier Science.

8. Ceppi, S., Gatti, N., \& Iuliano, C. (2011). Non-cooperative bargaining with arbitrary one-sided uncertainty. In Proceedings of the 13th workshop on agent-mediated electronic commerce.

9. Chatterjee, K., \& Samuelson, L. (1987). Bargaining with two-sided incomplete information: An infinite horizon model with alternating offers. Review of Economic Studies, 54(2), 175-192. 
10. Chatterjee, K., \& Samuelson, L. (1988). Bargaining under two-sided incomplete information: The unrestricted offers case. Operations Research, 36, 605-618.

11. Cramton, P. (1984). Bargaining with incomplete information: An infinite-horizon model with two-sided uncertainty. Review of Economic Studies, 51(4), 579-593.

12. Cramton, P. (1992). Strategic delay in bargaining with two-sided uncertainty. Review of Economic Studies, 59(1), 205-225.

13. Di Giunta, F., \& Gatti, N. (2006). Bargaining over multiple issues in finite horizon alternating-offers protocol. Annals of Mathematics and Artificial Intelligence, 47(3-4), 251-271.

14. Faratin, P., Sierra, C., \& Jennings, N. R. (1998). Negotiation decision functions for autonomous agents. International Journal of Robotics and Autonomous Systems, 24(3-4), 159-182.

15. Fatima, S. S., Wooldridge, M., \& Jennings, N. R. (2004). An agenda-based framework for multi-issue negotiation. Artificial Intelligence, 152(1), 1-45.

16. Fatima, S. S., Wooldridge, M., \& Jennings, N. R. (2006). Multi-issue negotiation with deadlines. Journal of Artificial Intelligence Research, 27, 381-417.

17. Fatima, S. S., Wooldridge, M., \& Jennings, N. R. (2006). On efficient procedures for multi-issue negotiation. In Proceedings of the trading agent design and analysis and agent mediated electronic commerce, pp. 71-84.

18. Fudenberg, D., \& Tirole, J. (1991). Game theory. Cambridge, MA: The MIT Press.

19. Gatti, N., Giunta, F. D., \& Marino, S. (2008). Alternating-offers bargaining with one-sided uncertain deadlines: An efficient algorithm. Artificial Intelligence, 172(8-9), 1119-1157.

20. Gatti, N., \& Iuliano, C. (2011). Computing an extensive-form perfect equilibrium in two-player games. In $A A A I$.

21. Gatti, N., \& Panozzo, F. (2012). New results on the verification of Nash refinements for extensive-form games. In Proceedings of the international joint conference on autonomous agents and multi-agent systems.

22. Gilpin, A., \& Sandholm, T. (2006). Finding equilibria in large sequential games of imperfect information. In Proceedings of the ACM conference on electronic commerce, pp. 160-169.

23. Gilpin, A., \& Sandholm, T. (2007). Lossless abstraction of imperfect information games. Journal of the ACM, 54(5), 25.

24. Gottlob, G., Greco, G., \& Scarcello, F. (2005). Pure Nash equilibria: Hard and easy games. Journal of Artificial Intelligence Research, 24, 357-406.

25. Jennings, N. R., Faratin, P., Lomuscio, A. R., Parsons, S., Sierra, C., \& Wooldridge, M. (2001). Automated negotiation: Prospects, methods and challenges. Group Decision and Negotiation, 10(2), 199-215.

26. Koller, D., Megiddo, N., \& von Stengel, B. (1994). Fast algorithms for finding randomized strategies in game trees. In Proceedings of the annual ACM symposium on theory of computing, pp. 750-759.

27. Kraus, S., Wilkenfeld, J., \& Zlotkin, G. (1995). Multiagent negotiation under time constraints. Artificial Intelligence, 75(2), 297-345.

28. Kreps, D., \& Wilson, R. (1982). Sequential equilibria. Econometrica, 50(4), 863-894.

29. Miltersen, P., \& Sorensen, T. (2006). Computing sequential equilibria for two-player games. In Proceedings of the ACM SIAM symposium on discrete algorithm (SODA), pp. 107-116.

30. Porter, R., Nudelman, E., \& Shoham, Y. (2008). Simple search methods for finding a Nash equilibrium. Games and Economic Behavior, 63, 642-662.

31. Rubinstein, A. (1982). Perfect equilibrium in a bargaining model. Econometrica, 50(1), 97-109.

32. Rubinstein, A. (1985). A bargaining model under incomplete information about time preferences. Econometrica, 53(5), 1151-1172.

33. Rubinstein, A. (1985). Choice of conjectures in a bargaining game with incomplete information. In Game-theoretic models of bargaining (pp. 99-114). Cambridge: Cambridge University Press.

34. Sandholm, T. (2000). Agents in electronic commerce: Component technologies for automated negotiation and coalition formation. Autonomous Agents and Multi-Agent Systems, 3(1), 73-96.

35. Sandholm, T., \& Vulkan, N. (1999). Bargaining with deadlines. In Proceedings of the national conference on artificial intelligence (AAAI), pp. 44-51.

36. Sim, K. M. (2002). A market-driven model for designing negotiation agents. Computational Intelligence, Special Issue in Agent Technology for E-Commerce, 18(4), 618-637.

37. Sim, K. M., \& Choi, C. Y. (2003). Agents that react to changing market situations. IEEE Transactions on Systems, Man and Cybernetics, Part B, 33(2), 188-201.

38. Stahl, I. (1972). Bargaining theory. Stockholm: Stockholm School of Economics. 